TRANSACTIONS OF THE

AMERICAN MATHEMATICAL SOCIETY

Volume 361, Number 11, November 2009, Pages 6115-6149

S 0002-9947(09)04905-8

Article electronically published on June 25, 2009

\title{
RATIONAL APPROXIMATIONS FOR VALUES OF DERIVATIVES OF THE GAMMA FUNCTION
}

\author{
TANGUY RIVOAL
}

\begin{abstract}
The arithmetic nature of Euler's constant $\gamma$ is still unknown and even getting good rational approximations to it is difficult. Recently, Aptekarev managed to find a third order linear recurrence with polynomial coefficients which admits two rational solutions $a_{n}$ and $b_{n}$ such that $a_{n} / b_{n}$ converges subexponentially to $\gamma$, viewed as $-\Gamma^{\prime}(1)$, where $\Gamma$ is the usual Gamma function. Although this is not yet enough to prove that $\gamma \notin \mathbb{Q}$, it is a major step in this direction.

In this paper, we present a different, but related, approach based on simultaneous Padé approximants to Euler's functions, from which we construct and study a new third order recurrence that produces a sequence in $\mathbb{Q}(z)$ whose height grows like the factorial and that converges sub-exponentially to $\log (z)+\gamma$ for any complex number $z \in \mathbb{C} \backslash(-\infty, 0]$, where $\log$ is defined by its principal branch. We also show how our approach yields in theory rational approximations of numbers related to $\Gamma^{(s)}(1)$ for any integer $s \geq 1$. In particular, we construct a sixth order recurrence which provides simultaneous rational approximations (of factorial height) converging sub-exponentially to the numbers $\gamma$ and $\Gamma^{\prime \prime}(1)-2 \Gamma^{\prime}(1)^{2}=\zeta(2)-\gamma^{2}$.
\end{abstract}

\section{INTRODUCTION}

The arithmetic nature of Euler's constant $\gamma$, defined as the limit of the sequence $s_{n}=\sum_{j=1}^{n} 1 / j-\ln (n+1)$, is still open. It is conjectured to be a transcendental number over $\mathbb{Q}$ but even its irrationality seems currently out of reach. A standard method for proving the irrationality of a classical constant $\alpha$ is to pull out of a hat or construct a sequence of rational approximations $\left(a_{n} / b_{n}\right)_{n \geq 0}$ such that $a_{n}, b_{n} \in \mathbb{Z}$ and $0<\left|b_{n} \alpha-a_{n}\right| \rightarrow 0$ as $n \rightarrow+\infty$. In fact, even getting a sequence of rationals $a_{n} / b_{n}$ whose height does not grow too fast and which converges fast to $\gamma$ is a difficult problem. It is easier to find averaging processes of the form $\sum_{k=0}^{n} a_{k, n}\left(\gamma-s_{k}\right)$ that converge geometrically to 0 , for some well-chosen integral weights $\left(a_{k, n}\right)_{0 \leq k \leq n}$ which are usually products of binomial coefficients (see [9]). But this does not help to study the arithmetic nature of $\gamma$.

For many classical constants, it turns out to be possible to construct "irrationality proving" rational approximations $a_{n} / b_{n}$ that satisfy a special property: both

Received by the editors February 28, 2008.

2000 Mathematics Subject Classification. Primary 11J13; Secondary 33C45, 33F10, 39A11.

Key words and phrases. Euler's constant, rational approximations, Padé approximants, linear recurrences, Birkhoff-Trjitzinsky theory. 
$\left(a_{n}\right)_{n \geq 0}$ and $\left(b_{n}\right)_{n \geq 0}$ are solutions of a linear recurrence of finite order with polynomial coefficients. For $e$, we can use the recurrence that generates its regular continued fraction, which was found by Euler. For $\ln (2), \zeta(2), \zeta(3)$, the best-known order is 2, leading to continued fractions but not the regular ones; these are the recurrences found by Apéry; see 1, 4]. For $\pi$, the best-known order is 3 (see [10, 12]).

For certain real algebraic numbers of Pisot type of degree $d$, it is possible to find approximations coming from a recurrence of order $d$, which generalises the recurrence of order 2 for real quadratic numbers coming from their continued fraction expansion. These approximations are constructed in [10, Sec. 6.1], although the recurrence is not written explicitly 1

It is also possible to find linear recurrences that produce rational approximations that converge fast to Catalan's constant $G$ (order 2, in [17, Sec. 9]), $\zeta(4)$ (order 2, in [8]) and $\zeta(5)$ (order 3, in [23]), but unfortunately, not sufficiently fast to prove irrationality. Except conjecturally $e$ and $\gamma$, all the above-mentioned numbers are periods in the sense of [13].

1.1. Aptekarev's approximations of $\gamma$. The existence of such a recurrence for $\gamma$ was unknown until recently when Aptekarev and his collaborators (in a series of papers [6]) found the third order recurrence

$$
\begin{aligned}
(16 n-15) & (n+1) u_{n+1}=\left(128 n^{3}+40 n^{2}-82 n-45\right) u_{n} \\
& -n\left(256 n^{3}-240 n^{2}+64 n-7\right) u_{n-1}+(16 n+1) n(n-1) u_{n-2},
\end{aligned}
$$

whose solutions $\left(p_{n}\right)_{n \geq 0},\left(q_{n}\right)_{n \geq 0}$ with $p_{0}=0, p_{1}=2, p_{2}=31 / 2$ and $q_{0}=1, q_{1}=$ $3, q_{2}=25$ are such that $q_{n} \in \mathbb{Z}, d_{n} p_{n} \in \mathbb{Z}\left(\right.$ where $\left.d_{n}=\operatorname{lcm}(1,2, \ldots, n)\right)$ and

$$
\left|\gamma-\frac{p_{n}}{q_{n}}\right| \sim c_{0} e^{-2 \sqrt{2 n}}, \quad\left|q_{n}\right| \sim \frac{c_{1}}{n^{1 / 4}} \frac{(2 n) !}{n !} e^{\sqrt{2 n}} .
$$

The exact asymptotic behaviors of both quantities in (1.2) are obtained in [6] via Birkhoff-Trjitzinsky's powerful theory of asymptotics of linear recurrences [7]. Unfortunately, the linear form $q_{n} \gamma-p_{n}$ does not tend to 0 and this is a fortiori the case for $d_{n}\left(q_{n} \gamma-p_{n}\right) \in \mathbb{Z} \gamma+\mathbb{Z}$. Hence, Aptekarev's approximations are not good enough to imply the irrationality of $\gamma$. But the existence of this recurrence is a surprising result of theoretical interest for our understanding of numbers which are conjecturally not periods, like $\gamma$.

The aim of this paper is to present an alternative construction to Aptekarev's which will enable us to produce both good rational functional approximations to the function $\ln (x)+\gamma$ for any $x>0$ and simultaneous rational approximations to $\gamma$ and $\zeta(2)-\gamma^{2}$.

1.2. Approximations of $\ln (x)+\gamma$. Let us fix any complex number $z \notin \frac{14}{3}+\frac{8}{3} \mathbb{N}$. Define two sequences $\left(P_{n}(z)\right)_{n \geq 0},\left(Q_{n}(z)\right)_{n \geq 0}$ of rational functions $\in \mathbb{Q}(z)$ by the

\footnotetext{
${ }^{1}$ This can be done by means of Proposition 5 in Section 5.1 starting from the recurrence (6.3) in [10, Sec. 6.1].
} 
following linear recurrence of order three:

$$
\begin{aligned}
(n+3)^{2}(3 z-8 n-14)(3 z-8 n-22) U_{n+3} & \\
=- & \left(24 n^{3}+7 z n^{2}+210 n^{2}-6 z^{2} n+56 z n+600 n\right. \\
\quad & \left.+96 z-15 z^{2}+564\right)(3 z-8 n-14) U_{n+2} \\
+\left(24 n^{3}-57 z n^{2}+\right. & 162 n^{2}+356 n+26 z^{2} n-258 z n \\
-3 z^{3}-285 z+59 z^{2} & +254)(3 z-8 n-30) U_{n+1} \\
& +(n+2)^{2}(3 z-8 n-22)(3 z-8 n-30) U_{n},
\end{aligned}
$$

with initial conditions

$$
\begin{aligned}
& P_{0}(z)=-1, P_{1}(z)=-9+16 z-3 z^{2}, P_{2}(z)=-19+27 z+\frac{27}{2} z^{2}-\frac{9}{4} z^{3}, \\
& Q_{0}(z)=2-z, Q_{1}(z)=4+4 z-z^{2}, Q_{2}(z)=6+27 z-\frac{1}{2} z^{3} .
\end{aligned}
$$

Since $z \notin \frac{14}{3}+\frac{8}{3} \mathbb{N}$, the leading coefficient of the recurrence is never 0 and this enables us to define a sequence $\left(U_{n}\right)_{n \geq 0}$ by its first three terms $U_{0}, U_{1}, U_{2}$. However, we will find alternative expressions of $P_{n}(z)$ and $Q_{n}(z)$ defined for any $z \in \mathbb{C}$.

Theorem 1. $(i)$ The solutions $\left(P_{n}(z)\right)_{n \geq 0}$ and $\left(Q_{n}(z)\right)_{n \geq 0}$ of the recurrence (1.3) are polynomials in $z$, of degree at most $n+1$. Furthermore, $n !^{2} P_{n}(z)$ and $n !^{2} Q_{n}(z)$ belong to $\mathbb{Z}[z]$.

(ii) For any real number $x>0$, there exist two constants $q(x) \neq 0$ and $s(x) \neq 0$ such that

$$
\left|Q_{n}(x)(\ln (x)+\gamma)-P_{n}(x)\right| \leq s(x) \exp \left(-3 / 2 x^{1 / 3} n^{2 / 3}+1 / 2 x^{2 / 3} n^{1 / 3}\right)
$$

and

$$
Q_{n}(x) \sim q(x) \exp \left(3 x^{1 / 3} n^{2 / 3}-x^{2 / 3} n^{1 / 3}\right)
$$

as $n \rightarrow+\infty$.

Furthermore, for any $x>0$, the sequence $Q_{n}(x)(\ln (x)+\gamma)-P_{n}(x)$ does not vanish for infinitely many integers $n$.

Remark 1. Our method shows that in fact $P_{n}(x) / Q_{n}(x)$ converges to $\log (x)+\gamma$ for any $x \in \mathbb{C} \backslash(-\infty, 0]$, with the principal branch for the logarithm. We did not try to obtain the exact speed of convergence but this could be done using the same tools.

It seems likely that it is possible to improve the estimate for the denominator of $Q_{n}(z)$.

Conjecture 1. For any $n \geq 0$, we have $n ! Q_{n}(z) \in \mathbb{Z}[z]$.

Theorem 1 implies that for any rational number $x=u / v>0$, there exists a constant $c(x) \neq 0$ and a sequence of rational numbers $\left(a_{n}(x) / b_{n}(x)\right)_{n \geq 0}$ such that the integers $a_{n}(x), b_{n}(x)$ satisfy

$$
\begin{aligned}
& \left|b_{n}(x)\right| \sim c(x) n !^{2} v^{n} \exp \left(3 x^{1 / 3} n^{2 / 3}-x^{2 / 3} n^{1 / 3}\right) \\
& \left|\ln (x)+\gamma-\frac{a_{n}(x)}{b_{n}(x)}\right|=\mathcal{O}\left(\exp \left(-9 / 2 x^{1 / 3} n^{2 / 3}+3 / 2 x^{2 / 3} n^{1 / 3}\right)\right)
\end{aligned}
$$

which is a good sub-exponential convergence, unfortunately not fast enough to imply the irrationality of $\ln (x)+\gamma$. Here are the recurrences for $x=1$ and $x=2$. 
Corollary 1. (i) The recurrence

$$
\begin{aligned}
& (n+3)^{2}(8 n+11)(8 n+19) U_{n+3}=\left(24 n^{2}+145 n+215\right)(8 n+11) U_{n+2} \\
& \quad-\left(24 n^{3}+105 n^{2}+124 n+25\right)(8 n+27) U_{n+1}+(n+2)^{2}(8 n+19)(8 n+27) U_{n}
\end{aligned}
$$

provides two sequences of rational numbers $\left(p_{n}\right)_{n \geq 0}$ and $\left(q_{n}\right)_{n \geq 0}$ with $p_{0}=-1$, $p_{1}=4, p_{2}=77 / 4$ and $q_{0}=1, q_{1}=7, q_{2}=65 / 2$ such that $\left(p_{n} / q_{n}\right)_{n \geq 0}$ converges to $\gamma$.

(ii) The recurrence

$$
\begin{aligned}
(n+1)(n+2)(n+3) U_{n+3}= & \left(3 n^{2}+19 n+29\right)(n+1) U_{n+2} \\
& -\left(3 n^{3}+6 n^{2}-7 n-13\right) U_{n+1}+(n+2)^{3} U_{n}
\end{aligned}
$$

provides two sequences of rational numbers $\left(p_{n}\right)_{n \geq 0}$ and $\left(q_{n}\right)_{n \geq 0}$ with $p_{0}=-1$, $p_{1}=11, p_{2}=71$ and $q_{0}=0, q_{1}=8, q_{2}=56$ such that $\left(p_{n} / q_{n}\right)_{n \geq 0}$ converges to $\ln (2)+\gamma$.

We also quote the following curious result, which is a consequence of the fact that $(\ln (x)+\gamma) Q_{n}(x) \rightarrow+\infty$ as $n \rightarrow+\infty$ for any real number $x>0$, except $x=e^{-\gamma}$.

Corollary 2. The only real number $x>0$ such that $\left(P_{n}(x)\right)_{n \geq 0}$ tends to 0 is $x=e^{-\gamma}$.

Numerically, it seems that there exists a sequence $\left(\alpha_{n}\right)_{n>0}$ of real algebraic numbers such that $P_{n}\left(\alpha_{n}\right)=0$ and $\alpha_{n} \rightarrow e^{-\gamma}$. A simple heuristic argument suggests that there exists a sequence $\left(\alpha_{n}\right)_{n \geq 0}$ of real algebraic numbers such that $P_{n}\left(\alpha_{n}\right)=0$ and

$$
\left|e^{-\gamma}-\alpha_{n}\right| \ll\left|\frac{P_{n}\left(e^{-\gamma}\right)}{Q_{n}\left(e^{-\gamma}\right)}\right|
$$

as $n \rightarrow+\infty$. Note that the right hand side is bounded by

$$
\exp \left(-9 e^{-\gamma} / 2 n^{2 / 3}(1+o(1))\right) \text {. }
$$

1.3. Simultaneous approximations of $\gamma$ and $\zeta(2)-\gamma^{2}$. The results above use the formula $\gamma=-\Gamma^{\prime}(1)$, where $\Gamma$ is the Gamma function. Our approach can in principle be generalised in order to study the number $\Gamma^{(s)}(1)$ for any integer $s \geq 1$. However, the details of the construction rapidly become very complicated and we present here an interesting result for $s=2$. We have not been able to find approximations for the number $\Gamma^{\prime \prime}(1)$ itself but only for the linear combination $\Gamma^{\prime \prime}(1)-2 \Gamma^{\prime}(1)^{2}=\zeta(2)-\gamma^{2}$.

Theorem 2. There exists a linear recurrence of order 6 , of the form

$$
\text { (1.6) } \begin{aligned}
& (n+6)^{4}(n+5)^{2}\left(83682825624 n^{19}+\cdots-79358069872714705024\right) U_{n+6} \\
= & (n+5)^{2}\left(502096953744 n^{23}+\cdots-1767373066831337541843456\right) U_{n+5} \\
& -\left(1255242384360 n^{25}+\cdots-77835199837793348629724160\right) U_{n+4} \\
& +\left(1673656512480 n^{25}+\cdots+2888284259604646368585600\right) U_{n+3} \\
& -\left(1255242384360 n^{25}+\cdots+80652658347913045992960000\right) U_{n+2} \\
+ & (n+3)^{2}\left(502096953744 n^{23}+\cdots+7635535812462194001878400\right) U_{n+1} \\
- & (n+3)^{2}(n+2)^{4}\left(83682825624 n^{19}+\cdots+15621570330176916950400\right) U_{n},
\end{aligned}
$$


whose coefficients are polynomials of degree 25 with integer coefficients, which has three solutions $\left(a_{1, n}\right)_{n \geq 0},\left(a_{2, n}\right)_{n \geq 0}$ and $\left(b_{n}\right)_{n \geq 0}$ satisfying the following properties: for all $n \geq 0, a_{1, n}, a_{2, n}$ and $b_{n}$ belong to $\frac{1}{(3 n) !(3 n+2) !} \mathbb{Z}$, and we have

$$
\left|\gamma-\frac{a_{1, n}}{b_{n}}\right| \ll \frac{1}{n^{3 / 8} b_{n}},\left|\zeta(2)-\gamma^{2}-\frac{a_{2, n}}{b_{n}}\right| \ll \frac{1}{n^{3 / 8} b_{n}},
$$

and

$$
\left|b_{n}\right| \sim \frac{c_{1}}{n^{3 / 8}} \exp \left(4 \sqrt{2} n^{3 / 4}-5 \sqrt{2} / 8 n^{1 / 4}\right),
$$

for some constant $c_{1}>0$, as $n \rightarrow+\infty$.

As it is already clear, the coefficients of the recurrence are huge and the reader can find them in the Annex, together with the initial values of the sequences $\left(a_{1, n}\right)_{n \geq 0}$, $\left(a_{2, n}\right)_{n \geq 0}$ and $\left(b_{n}\right)_{n \geq 0}$. Explicit expressions for these sequences can be found in Section 9. The denominator found for $a_{1, n}, a_{2, n}$ and $b_{n}$ is probably not optimal. In particular, we propose the following conjecture.

Conjecture 2. For any $n \geq 0$, we have $n !^{2} b_{n} \in \mathbb{Z}$.

Since the sequences $Q_{n}(z)$ in Theorem 11 and $b_{n}$ in Theorem 2 are constructed in a very similar way, Conjectures 1 and 2 suggest the existence of a very general "Denominators Conjecture" behind them. Although proving these conjectures will not be sufficient to prove the irrationality of $\gamma$ or $\zeta(2)-\gamma^{2}$, they might be worth pursuing to get closer to such results. Moreover, it would be of great interest to find a linear recurrence of order 2 yielding good rational approximations to $\gamma$.

The proofs of our theorems are quite involved and we have to split them into several parts. In the next section, we provide an overview of the method, which is simple in spirit.

\section{Overview OF THE METHOD}

There exist many different expressions for Euler's constant and until recently it wasn't clear which one is useful. One such expression is

$$
\int_{0}^{\infty} \ln (t) e^{-t} \mathrm{~d} t=\Gamma^{\prime}(1)=-\gamma
$$

where $\Gamma$ denotes the usual Gamma function. More generally, for any $n \geq 0$, we have

$$
\int_{0}^{\infty} t^{n} \ln (t) e^{-t} \mathrm{~d} t=\Gamma^{\prime}(n+1)=n !\left(H_{n}-\gamma\right),
$$

where $H_{n}=\sum_{j=1}^{n} 1 / j$. Hence,

$$
\int_{0}^{\infty}\left(\sum_{k=0}^{n} a_{k} t^{k}\right) \ln (t) e^{-t} \mathrm{~d} t=\sum_{k=0}^{n} a_{k} k !\left(H_{k}-\gamma\right) \in \mathbb{Q}+\mathbb{Q} \gamma,
$$

provided the $a_{k}$ 's are rational numbers.

Hence, it remains to find a special sequence of polynomials $\sum_{k=0}^{n} a_{k} t^{k}$ such that the linear form given by (2.2) yields good rational approximations to $\gamma$. Aptekarev chose

$$
\mathbf{P}_{n}(t)=\frac{e^{t}}{n !^{2}(1-t)}\left(t^{n}\left(t^{n}(1-t)^{2 n+1} e^{-t}\right)^{(n)}\right)^{(n)} \in \mathbb{Q}[t]
$$


for reasons related to the construction of simultaneous Padé approximants of type II at $z=\infty$ to the functions

$$
\int_{0}^{1} \frac{(1-t) \ln (t) e^{-t}}{z-t} \mathrm{~d} t, \quad \int_{1}^{\infty} \frac{(1-t) \ln (t) e^{-t}}{z-t} \mathrm{~d} t
$$

and two other similar functions without the factor $\ln (t)$ in the integrand. Two of the four remainders of these approximants are given by

$$
\int_{0}^{1} \frac{\mathbf{P}_{n}(t)}{z-t}(1-t) \ln (t) e^{-t} \mathrm{~d} t, \quad \int_{1}^{\infty} \frac{\mathbf{P}_{n}(t)}{z-t}(1-t) \ln (t) e^{-t} \mathrm{~d} t .
$$

These integrals are defined a priori only for $z \notin[0,+\infty)$, but they also converge for $z=1$. In a certain sense (see [6] for details), this suggests that their sum $s_{n}$ at $z=1$, given by

$$
\int_{0}^{1} \mathbf{P}_{n}(t) \ln (t) e^{-t} \mathrm{~d} t+\int_{1}^{\infty} \mathbf{P}_{n}(t) \ln (t) e^{-t} \mathrm{~d} t=\int_{0}^{\infty} \mathbf{P}_{n}(t) \ln (t) e^{-t} \mathrm{~d} t
$$

has good approximation properties. By (2.2), we have $s_{n}=q_{n} \gamma-p_{n}$ for some rational numbers $p_{n}, q_{n}$ and it turns out that $\left(p_{n}\right)_{n \geq 0},\left(q_{n}\right)_{n \geq 0}$ and $\left(s_{n}\right)_{n \geq 0}$ all satisfy Aptekarev's recurrence (1.1).

Our use of (2.2) in Theorem 1 is less obvious but still underlies the construction presented in this paper, which is also based on certain Padé approximants of type II at $z=\infty$ but for the functions

$$
\mathcal{E}_{1}(z)=\int_{0}^{\infty} \frac{e^{-t}}{z-t} \mathrm{~d} t \quad \text { and } \quad \mathcal{E}_{2}(z)=\int_{0}^{\infty} \frac{\ln (t) e^{-t}}{z-t} \mathrm{~d} t .
$$

Note that $\mathcal{E}_{2}(z)$ is defined at $z=1$ but not $\mathcal{E}_{1}(z)$. Consider the polynomial $A_{n}$ of degree $2 n$ defined by $A_{n}(z)=\frac{1}{n !^{2}} e^{z}\left(z^{n}\left(z^{n} e^{-z}\right)^{(n)}\right)^{(n)} \in \mathbb{Q}[z]$, which will initially play a similar role to $\mathbf{P}_{n}$ above. Without going into details, which will be progressively given in the next sections, we will first find two other sequences of polynomials $B_{n}(z) \in \mathbb{Q}[z]$ and $C_{n}(z) \in \mathbb{R}[z]$ of degree at most $2 n$ such that the remainders of these approximants are given by

$$
R_{1, n}(z)=\int_{0}^{\infty} \frac{A_{n}(t)}{z-t} e^{-t} \mathrm{~d} t=A_{n}(z) \mathcal{E}_{1}(z)+B_{n}(z)=\mathcal{O}\left(z^{-n-1}\right)
$$

and

$$
R_{2, n}(z)=\int_{0}^{\infty} \frac{A_{n}(t)}{z-t} \ln (t) e^{-t} \mathrm{~d} t=A_{n}(z) \mathcal{E}_{2}(z)+C_{n}(z)=\mathcal{O}\left(z^{-n-1}\right) .
$$

A crucial property is that we can write $C_{n}(z)=-B_{n}(z) \gamma-D_{n}(z)$ where $D_{n}(z) \in$ $\mathbb{Q}[z]$. Hence $R_{2, n}(z)=A_{n}(z) \mathcal{E}_{2}(z)-B_{n}(z) \gamma-D_{n}(z)$. The fact that $A_{n}(z)$ and $B_{n}(z)$ occur in both remainder functions enables us to deal with $\ln (x)+\gamma$ for any $x>0$ in Theorem 1 rather than just with $\gamma$.

To isolate $\gamma$ when $x=1$, it suffices to cancel out $\mathcal{E}_{2}(1)$ by considering the determinant $S_{n}(1)=A_{n}(1) R_{2, n+1}(1)-A_{n+1}(1) R_{2, n}(1) \in \mathbb{Q}+\mathbb{Q} \gamma$. In such contexts, quantities such as $S_{n}(1)$ do not always have good properties but this is the case here; i.e., $S_{n}(1)$ provides good rational approximations of $\gamma$. Furthermore, $\left(S_{n}(1)\right)_{n \geq 0}$ satisfies the recurrence (1.4). A "higher dimensional" generalisation of these Padé approximants gives Theorem 2 


\section{EXPRESSION OF $\Gamma^{(s)}(n)$}

In this short section, we recall some basic facts about the values of the derivatives of the Gamma function at the point 1 . The identity

$$
\Gamma(z+1)=\exp \left(-\gamma z+\sum_{k=2}^{\infty} \frac{(-1)^{k} \zeta(k)}{k} z^{k}\right) \quad(|z|<1)
$$

follows immediately from the classical product expansion of $\Gamma$ (see 3 , p. 3, Theorem 1.1.2]). It shows that

$$
\Gamma^{(s)}(1)=\sum_{k_{1}+2 k_{2}+\cdots+s k_{s}=s} g_{k_{1}, \ldots, k_{s}} \gamma^{k_{1}} \zeta(2)^{k_{2}} \cdots \zeta(s)^{k_{s}}
$$

for some $g_{k_{1}, \ldots, k_{s}} \in \mathbb{Q}$. For example, $\Gamma^{\prime}(1)=-\gamma, \Gamma^{\prime \prime}(1)=\zeta(2)+\gamma^{2}, \Gamma^{\prime \prime \prime}(1)=$ $-2 \zeta(3)-3 \gamma \zeta(2)-\gamma^{3}$.

We remark that the formula (3.1) is coherent with the folklore principle that if we add $\gamma$ to the hierarchy of multiple zeta values or periods, then its weight should be set to 1: this is natural because $\gamma$ is in a certain sense a regularised value of the divergent series $\zeta(1)$ and the weight of $\zeta(s)$ is $s$ for any integer $s \geq 2$. See [19, p. 588] for details.

Let us define $H_{n}^{[s]}$ recursively by $H_{n}^{[s]}=s \sum_{i=1}^{n} \frac{1}{i} H_{i-1}^{[s-1]}$, with $H_{n}^{[0]}=1$ for any $n \geq 0$ and $H_{0}^{[s]}=0$ for any $s \geq 1$. In an expanded form, we have

$$
H_{n}^{[s]}=s ! \sum_{1 \leq i_{1}<\cdots<i_{s} \leq n} \frac{1}{i_{1} i_{2} \cdots i_{s}}
$$

for $s \geq 1$.

Proposition 1. For any integers $n \geq 0$ and $s \geq 0$, we have

$$
\int_{0}^{\infty} t^{n} \ln (t)^{s} e^{-t} \mathrm{~d} t=\Gamma^{(s)}(n+1)=n ! \sum_{j=0}^{s}\left(\begin{array}{l}
s \\
j
\end{array}\right) H_{n}^{[s-j]} \Gamma^{(j)}(1),
$$

which is a linear form in $1, \Gamma^{(1)}(1), \ldots, \Gamma^{(s)}(1)$ with integer coefficients.

In particular, for $s=1$, we recover (2.1) and for $s=2$, we get

$$
\int_{0}^{\infty} t^{n} \ln (t)^{2} e^{-t} \mathrm{~d} t=n !\left(\zeta(2)+\gamma^{2}-2 H_{n} \gamma+H_{n}^{[2]}\right) .
$$

Proof. We proceed by induction on $n \geq 0$. The formula is true for $n=0$ with the conventions adopted. Assume it is true for some $n$ and let us prove it for $n+1$. The functional equation $\Gamma(z+1)=z \Gamma(z)$ implies that $\Gamma^{(s)}(n+1)=n \Gamma^{(s)}(n)+s \Gamma^{(s-1)}(n)$. Using the induction, we deduce that

$$
\begin{aligned}
\Gamma^{(s)}(n+1) & =(n-1) ! n \sum_{j=0}^{s}\left(\begin{array}{c}
s \\
j
\end{array}\right) H_{n-1}^{[s-j]} \Gamma^{(j)}(1)+(n-1) ! s \sum_{j=0}^{s-1}\left(\begin{array}{c}
s-1 \\
j
\end{array}\right) H_{n-1}^{[s-j-1]} \Gamma^{(j)}(1) \\
& =n ! \Gamma^{(s)}(1)+n ! \sum_{j=0}^{s-1}\left(\begin{array}{c}
s \\
j
\end{array}\right) \Gamma^{(j)}(1)\left(H_{n-1}^{[s-j]}+\frac{s-j}{n} H_{n-1}^{[s-j-1]}\right) \\
& =n ! \sum_{j=0}^{s}\left(\begin{array}{c}
s \\
j
\end{array}\right) H_{n}^{[s-j]} \Gamma^{(j)}(1),
\end{aligned}
$$


where we have used the trivial fact that $H_{n-1}^{[s-j]}+\frac{s-j}{n} H_{n-1}^{[s-j-1]}=H_{n}^{[s-j]}$ for $j<s$ to simplify the sum. This proves the formula for $n+1$ and finishes the induction.

The assertion concerning the integrality of the coefficients $n !\left(\begin{array}{l}s \\ j\end{array}\right) H_{n}^{[s-j]}$ is a consequence of the property

$$
n ! \sum_{1 \leq i_{1}<\cdots<i_{s} \leq n} \frac{1}{i_{1} i_{2} \cdots i_{s}} \in \mathbb{N}
$$

which is obvious.

\section{EULER's $\mathcal{E}$-FUNCTIONS}

In this section, we study Euler's functions, whose Taylor coefficients at infinity are related to derivatives of the Gamma function at the point 1 . We also present a construction of certain simultaneous Padé approximants to these functions, which will be at the heart of Theorems 1 and 2 ,

4.1. Asymptotic expansion of Euler's functions. From now on, the argument of a complex number $z$ is such that $-\pi \leq \arg (z)<\pi$, and thus the logarithm is defined with its principal branch.

The construction of our approximations to the numbers $\ln (x)+\gamma$ is based on certain properties of Euler functions $\mathcal{E}_{s}$ defined 2 by

$$
\mathcal{E}_{s}(z)=\int_{0}^{\infty} \frac{\ln (t)^{s-1} e^{-t}}{z-t} \mathrm{~d} t
$$

(where $s \geq 1$ is an integer), which are analytic functions of $z$ in $\mathbb{C} \backslash[0,+\infty)$.

Proposition 2. For any integer $s \geq 1$, the following asymptotic expansion holds in any angular sector of vertex 0 that does not contain $[0,+\infty)$ :

$$
\mathcal{E}_{s}(z) \sim \widehat{\mathcal{E}}_{s}(z)=\sum_{k=1}^{\infty} \frac{\Gamma^{(s-1)}(k)}{z^{k}} .
$$

Proof. For any fixed integer $n \geq 0$, we have

$$
z^{n} \mathcal{E}_{s}(z)=\int_{0}^{\infty} \frac{z^{n}-t^{n}}{z-t} \ln (t)^{s-1} e^{-t} \mathrm{~d} t+\int_{0}^{\infty} \frac{t^{n}}{z-t} \ln (t)^{s-1} e^{-t} \mathrm{~d} t
$$

The first integral is easily computed:

$$
\begin{aligned}
\int_{0}^{\infty} \frac{z^{n}-t^{n}}{z-t} \ln (t)^{s-1} e^{-t} \mathrm{~d} t & =\sum_{k=1}^{n} z^{n-k} \int_{0}^{\infty} t^{k-1} \ln (t)^{s-1} e^{-t} \mathrm{~d} t \\
& =\sum_{k=1}^{n} \Gamma^{(s-1)}(k) z^{n-k}
\end{aligned}
$$

The second integral is $\mathcal{O}(1 / z)$ as $z \rightarrow \infty$ in a suitable angular sector because

$$
\int_{0}^{\infty}\left|t^{n} \ln (t)^{s-1} e^{-t}\right| \mathrm{d} t<+\infty
$$

The $\mathcal{O}$ depends on $n$ and $s$.

\footnotetext{
${ }^{2}$ The function $\mathcal{E}_{1}$ was used by Euler in his famous attempt at summing the divergent series $\sum_{n=1}^{\infty}(-1)^{n} n$ ! as $\mathcal{E}_{1}(-1)$; see [11, p. 26].
} 
Hence, it follows that, for any fixed $n \geq 0$,

$$
\mathcal{E}_{s}(z)=\sum_{k=1}^{n} \frac{\Gamma^{(s-1)}(k)}{z^{k}}+\mathcal{O}\left(\frac{1}{z^{n+1}}\right)
$$

as $z \rightarrow \infty$ in a suitable angular sector, where the $\mathcal{O}$ depends on $n, s$. We have thus obtained the asymptotic expansion of $\mathcal{E}_{s}(z)$ in Poincaré's sense.

Remark 2. It follows from (3.2) that $\int_{0}^{\infty}\left|t^{n} \ln (t)^{s-1} e^{-t}\right| \mathrm{d} t \ll \log (n)^{s-1} n$ !. Hence, the asymptotic expansion is Gevrey of order 1, following a standard terminology [15].

We will need later the fact that $\mathcal{E}_{1}(z) \notin \mathbb{C}(z)$. This can be proved by showing that the differential equation $y^{\prime}(z)+y(z)=1 / z$ satisfied by $\mathcal{E}_{1}(z)$ has no solution in $\mathbb{C}(z)$ : assume that $R(z) \in \mathbb{C}(z)$ is such a solution and obtain a contradiction using its Laurent series at $z=0$.

4.2. Multiple orthogonal polynomials of Laguerre-type. Laguerre polynomials $L_{n}(z)$ are defined by $L_{n}(z)=\frac{1}{n !} e^{z}\left(z^{n} e^{-z}\right)^{(n)}$ for any integer $n \geq 0$. They are of degree $n$ and orthogonal on $[0,+\infty)$ with respect to the weight $e^{-t}$; i.e., for any integer $k \in\{0,1, \ldots, n-1\}$, we have

$$
\int_{0}^{\infty} t^{k} L_{n}(t) e^{-t} \mathrm{~d} t=0 .
$$

We now define a generalisation $\left(A_{s, n}(z)\right)_{n \geq 0}$ (for any integer $s \geq 1$ ) of Laguerre polynomials by $A_{0, n}(z) \equiv 1$ and, recursively, $A_{r+1, n}(z)=\frac{1}{n !} e^{z}\left(z^{n} e^{-z} A_{r, n}(z)\right)^{(n)}$. In particular, $A_{1, n}(z)=L_{n}(z)$ and (using induction on $s$ ) we find that the degree of $A_{s, n}$ is $s n$. The main property of these polynomials is the following.

Proposition 3. For any integer $s \geq 1$, the sequence $\left(A_{s, n}\right)_{n \geq 0}$ is a sequence of multiple orthogonal polynomials on $[0,+\infty)$ with respect to the weights $e^{-t}, \ln (t) e^{-t}, \ldots$, $\ln (t)^{s-1} e^{-t}$; i.e., for any integers $k \in\{0,1, \ldots, n-1\}$ and $j \in\{1, \ldots, s\}$, we have

$$
\int_{0}^{\infty} t^{k} A_{s, n}(t) \ln (t)^{j-1} e^{-t} \mathrm{~d} t=0
$$

Proof. By induction on $k$ and $s$, using repeated integrations by parts.

4.3. Padé approximants of type II. By a standard method, the sequence of multiple orthogonal polynomials $\left(A_{s, n}(x)\right)_{n \geq 0}$ enables us to construct the simultaneous Padé approximants $[s n ; s n, \ldots, s n]$ of type II at $z=\infty$ (in the asymptotic sense) of the family of functions $\left(\mathcal{E}_{j}(z)\right)_{j=1, \ldots, s} 3$

Indeed, let us define the polynomials

$$
B_{j, s, n}(z)=\int_{0}^{\infty} \frac{A_{s, n}(t)-A_{s, n}(z)}{z-t} \ln (t)^{j-1} e^{-t} \mathrm{~d} t \in \mathbb{R}[z]
$$

and the remainder functions

$$
R_{j, s, n}(z)=\int_{0}^{\infty} \frac{A_{s, n}(t)}{z-t} \ln (t)^{j-1} e^{-t} \mathrm{~d} t .
$$

Then, for any $n \geq 0$ and any $j=1, \ldots, s$, we have

$$
R_{j, s, n}(z)=A_{s, n}(z) \mathcal{E}_{j}(z)+B_{j, s, n}(z)=\mathcal{O}\left(z^{-n-1}\right),
$$

\footnotetext{
${ }^{3}$ These asymptotic Padé approximants give the Padé approximants of type II in the usual sense for the family of formal series $\left(\widehat{\mathcal{E}}_{j}(x)\right)_{j=1, \ldots, s}$; see [16] Sec. 2] for details on this point.
} 
where $\mathcal{O}\left(z^{-n-1}\right)$ means that the asymptotic expansion of $R_{j, s, n}(z)$ at infinity starts at $n+1$. This is a consequence of the following proposition, which is more precise and which will be used later.

Proposition 4. For any integers $n \geq 0, s \geq 1, j=1, \ldots, s$, the asymptotic expansion of $R_{j, s, n}(z)$ in any angular sector of vertex 0 that does not contain $[0,+\infty)$ is given by

$$
R_{j, s, n}(z) \sim(-1)^{n s} \sum_{k=1}^{\infty} \frac{1}{z^{k}} \frac{\partial^{j-1}}{\partial k^{j-1}}\left(\frac{(k-n)_{n}^{s}}{n !^{s}} \Gamma(k)\right)
$$

where by definition, $(\alpha)_{0}=1$ and $(\alpha)_{m}=\alpha(\alpha+1) \cdots(\alpha+m-1)$ for $m \geq 1$.

Proof. We mimic the proof of Proposition 2 For any fixed integer $k \geq 0$, we have

$$
z^{k} R_{j, s, n}(z)=\int_{0}^{\infty} A_{s, n}(t) \frac{z^{k}-t^{k}}{z-t} \ln (t)^{j-1} e^{-t} \mathrm{~d} t+\int_{0}^{\infty} \frac{A_{s, n}(t) t^{k}}{z-t} \ln (t)^{j-1} e^{-t} \mathrm{~d} t
$$

The second integral is $\mathcal{O}(1 / z)$ as $z \rightarrow \infty$ in a suitable angular sector because

$$
\int_{0}^{\infty}\left|A_{s, n}(t) t^{k} \ln (t)^{j-1} e^{-t}\right| \mathrm{d} t<+\infty .
$$

It remains to compute the first integral. For this, we remark that

$$
\begin{aligned}
\int_{0}^{\infty} A_{s, n}(t) \frac{z^{k}-t^{k}}{z-t} \ln (t)^{j-1} e^{-t} \mathrm{~d} t & =\sum_{\ell=1}^{k} z^{k-\ell} \int_{0}^{\infty} t^{\ell-1} A_{s, n}(t) \ln (t)^{j-1} e^{-t} \mathrm{~d} t \\
& =\sum_{\ell=1}^{k} z^{k-\ell} \int_{0}^{\infty} \frac{\partial^{j-1} t^{\ell+\alpha-1}}{\partial \alpha^{j-1}}{ }_{\mid \alpha=0} A_{s, n}(t) e^{-t} \mathrm{~d} t \\
& =\sum_{\ell=1}^{k} z^{k-\ell} \frac{\partial^{j-1}}{\partial \alpha^{j-1}}\left(\int_{0}^{\infty} t^{\ell+\alpha-1} A_{s, n}(t) e^{-t} \mathrm{~d} t\right)_{\mid \alpha=0}
\end{aligned}
$$

where the change in the order of integration and derivation is justified. By repeated integrations by parts, we get that for any real number $v>0$, we have

$$
\int_{0}^{\infty} t^{v-1} A_{s, n}(t) e^{-t} \mathrm{~d} t=(-1)^{n s} \frac{(v-n)_{n}^{s}}{n !^{s}} \Gamma(v) .
$$

Hence, it follows that, for any fixed $k \geq 0$,

$$
R_{j, s, n}(z)=(-1)^{n s} \sum_{\ell=1}^{k} \frac{1}{z^{\ell}} \frac{\partial^{j-1}}{\partial \alpha^{j-1}}\left(\frac{(\ell+\alpha-n)_{n}^{s}}{n !^{s}} \Gamma(\ell+\alpha)\right)_{\mid \alpha=0}+\mathcal{O}\left(\frac{1}{z^{k+1}}\right)
$$

as $z \rightarrow \infty$ in a suitable angular sector, where the $\mathcal{O}$ depends on $j, k, n, s$. We have thus obtained the asymptotic expansion of $R_{j, s, n}(z)$ in Poincaré's sense.

Let us conclude this section with a simple but important remark. Using (3.2), we see immediately that there exist polynomials $\widetilde{B}_{k, s, n}(z) \in \mathbb{Q}[z], k=1, \ldots, s$, such that for any $j=1, \ldots, s$,

$$
B_{j, s, n}(z)=\sum_{k=1}^{j}\left(\begin{array}{l}
j-1 \\
k-1
\end{array}\right) \Gamma^{(j-k)}(1) \widetilde{B}_{k, s, n}(z) .
$$

In particular, $\widetilde{B}_{1, s, n}(z)=B_{1, s, n}(z)$. 


\section{Some PROPERTIES OF LINEAR RECURRENCES}

In this section, we collect some general results on linear recurrences of finite order that will be used at many different steps in the next sections.

5.1. "Determinantal" recurrences. Let us fix an integer $d \geq 1$. We define a linear recurrence of order $d+1$ by

$$
U_{n+d+1}=\sum_{k=0}^{d} a_{k, n} U_{n+k},
$$

where the $a_{k, n}$ 's are complex numbers. For reasons that will be clear later, given $d$ solutions $\left(U_{\ell, n}\right)_{n \geq 0}, \ell=1, \ldots, d$, of (5.1), it is interesting to consider the sequence of determinants

$$
V_{n}=\left|\begin{array}{cccc}
U_{1, n} & U_{1, n+1} & \ldots & U_{1, n+d-1} \\
U_{2, n} & U_{2, n+1} & \ldots & U_{2, n+d-1} \\
\vdots & \vdots & \vdots & \vdots \\
U_{d, n} & U_{d, n+1} & \ldots & U_{d, n+d-1}
\end{array}\right| .
$$

We want to show that $\left(V_{n}\right)_{n \geq 0}$ is also a solution of a recurrence of order $d+1$ with coefficients that can be made explicit.

Proposition 5. The sequence $\left(V_{n}\right)_{n \geq 0}$ is a solution of the linear recurrence of order $d+1$ :

$$
V_{n+d+1}=\sum_{k=0}^{d} b_{k, n} V_{n+k},
$$

where $b_{0, n}=\prod_{\ell=0}^{d-1} a_{0, n+\ell}$ and $b_{j, n}=(-1)^{d j+1} a_{d-j+1, n+j-1} \prod_{\ell=j}^{d-1} a_{0, n+\ell}, \quad j=$ $1, \ldots, d$. (For $j=d$, the value of the empty product is set to 1.)

Remark 3. In this paper, we will need only the case $d=2$ (recurrence of order 3), but the proof is not more difficult in the general case. This case is as follows: the sequence of determinants $\left(V_{n}\right)_{n \geq 0}$ associated to $U_{n+3}=p_{n} U_{n+2}+q_{n} U_{n+1}+r_{n} U_{n}$ satisfies the recurrence

$$
V_{n+3}=-q_{n+1} V_{n+2}-p_{n} r_{n+1} V_{n+1}+r_{n} r_{n+1} V_{n}
$$

Sketch of proof. Let us introduce the determinants

$$
V_{j, n}=\left|\begin{array}{cccccc}
U_{1, n} & \ldots & U_{1, n+d-j} & U_{1, n+d-j+2} & \ldots & U_{1, n+d} \\
U_{2, n} & \ldots & U_{2, n+d-j} & U_{2, n+d-j+2} & \ldots & U_{2, n+d} \\
\vdots & \vdots & \vdots & \vdots & \vdots & \vdots \\
U_{d, n} & \ldots & U_{d, n+d-j} & U_{d, n+d-j+2} & \ldots & U_{d, n+d}
\end{array}\right|
$$

for $j=1, \ldots, d$, so that $V_{1, n}=V_{n}$.

Consider the determinant $a_{0, n} V_{j, n}$, where $a_{0, n}$ is put as a factor of the first column. In the latter, we replace each $a_{0, n} U_{\ell, n}$ by $U_{\ell, n+d+1}-\sum_{k=1}^{d} a_{k, n} U_{\ell, n+k}$. By multilinearity, we expand the resulting determinant with respect to the first column: we end up with a sum of two determinants only, which can be rewritten as

$$
a_{0, n} V_{j, n}=(-1)^{d+1} V_{j+1, n+1}+(-1)^{d-j+1} a_{d-j+1, n} V_{1, n+1},
$$

for any $n \geq 0$ and $j=1, \ldots, d$, with the convention that $V_{d+1, n+1}=V_{1, n+2}$. 
If we now replace $n$ by $n+j-1$ in (5.4), we get the system $V_{j+1, n+j}=(-1)^{d+1} a_{0, n+j-1} V_{j, n+j-1}+(-1)^{j+1} a_{d-j+1, n+j-1} V_{1, n+j}, \quad j=1, \ldots, d$, from which, after some tedious computations, we can express $V_{1, n+d+1}$ as a linear form in $V_{1, n+d}, V_{1, n+d-1}, \ldots, V_{1, n}$, whose coefficients are exactly the $b_{k, n}$ 's.

We will also need to find explicitly the recurrence satisfied by a determinant formed from two solutions of a recurrence of order 4 .

Proposition 6. Consider two solutions $\left(A_{n}\right)_{n>0}$ and $\left(B_{n}\right)_{n>0}$ of the recurrence $U_{n+4}=s_{n} U_{n+3}+p_{n} U_{n+2}+q_{n} U_{n+1}+r_{n} U_{n}$

Then, the sequence of determinants $\left(A_{n} B_{n+1}-A_{n+1} B_{n}\right)_{n \geq 0}$ satisfies a recurrence of order 6 :

$$
a_{n} V_{n+6}+b_{n} V_{n+5}+c_{n} V_{n+4}+d_{n} V_{n+3}+e_{n} V_{n+2}+f_{n} V_{n+1}+g_{n} V_{n}=0,
$$

where

$$
\begin{gathered}
a_{n}=q_{n+2} q_{n+1}+r_{n+2} s_{n+1} s_{n}, \\
b_{n}=r_{n+2} s_{n+1} p_{n+3} s_{n}-r_{n+3} s_{n+2} q_{n+1}+q_{n+3} r_{n+2} s_{n}+p_{n+3} q_{n+2} q_{n+1}, \\
c_{n}=-r_{n+3} s_{n+2} p_{n+2} q_{n+1}+r_{n+2} s_{n+1} r_{n+3} s_{n+2}+p_{n+2} q_{n+3} r_{n+2} s_{n} \\
+r_{n+2} q_{n+2} q_{n+3}+q_{n+3} s_{n+2} q_{n+2} q_{n+1}+r_{n+2} s_{n+1} q_{n+3} s_{n+2} s_{n}, \\
d_{n}=r_{n+3} p_{n+2} q_{n+2} q_{n+1}-q_{n+3} q_{n+2}^{2} q_{n+1}+r_{n+2} s_{n} s_{n+1}^{2} r_{n+3} s_{n+2} \\
+r_{n+2} s_{n+1} p_{n+1} r_{n+3} s_{n+2}+s_{n+1} r_{n+3} p_{n+2} r_{n+2} s_{n}+r_{n+2} p_{n+1} q_{n+2} q_{n+3}, \\
e_{n}=-r_{n+2}\left(q_{n+2} q_{n+1} s_{n} q_{n+3}-r_{n+2} p_{n+1} q_{n+3} s_{n}+p_{n+1} q_{n+1} r_{n+3} s_{n+2}\right. \\
\left.+s_{n+1} r_{n+3} r_{n+2} s_{n}+s_{n+1} s_{n} q_{n+1} r_{n+3} s_{n+2}+r_{n+3} q_{n+2} q_{n+1}\right), \\
f_{n}=-r_{n+2} r_{n+1}\left(q_{n+3} r_{n+2} s_{n}-r_{n+3} s_{n+2} q_{n+1}-q_{n+2} p_{n} q_{n+3}-s_{n+1} p_{n} r_{n+3} s_{n+2}\right), \\
g_{n}=-r_{n+1} r_{n+2} r_{n}\left(q_{n+2} q_{n+3}+s_{n+1} r_{n+3} s_{n+2}\right) .
\end{gathered}
$$

Remark 4. There are four independent solutions for a recurrence of order 4; hence six different determinants can be constructed on two solutions. It is therefore natural that we get a linear recurrence of order 6 .

More generally, given a linear recurrence of order $s$ and an integer $t \in\{1, \ldots, s\}$, there are $\left(\begin{array}{l}s \\ t\end{array}\right)$ different determinants $\operatorname{det}\left(u_{j, n+i-1}\right)_{\substack{i=1, \ldots, t \\ j=1, \ldots, t}}$, where the $\left(u_{j, n}\right)_{n \geq 0}$ are solutions of the recurrence. Propositions 5 and 6 suggest that these determinants satisfy a linear recurrence of order $\left(\begin{array}{l}s \\ t\end{array}\right)$.

Sketch of proof. Let

$$
X_{n}=\left|\begin{array}{ll}
A_{n} & A_{n+1} \\
B_{n} & B_{n+1}
\end{array}\right|, Y_{n}=\left|\begin{array}{cc}
A_{n} & A_{n+2} \\
B_{n} & B_{n+2}
\end{array}\right|, Z_{n}=\left|\begin{array}{cc}
A_{n} & A_{n+3} \\
B_{n} & B_{n+3}
\end{array}\right| .
$$

We want to show that $\left(X_{n}\right)_{n \geq 0}$ is a solution of (5.5).

Using the recurrence satisfied by $\left(A_{n}\right)_{n \geq 0}$ and $\left(B_{n}\right)_{n \geq 0}$, we find that

$$
\left\{\begin{array}{l}
r_{n-1} X_{n-1}=-Z_{n}+s_{n-1} Y_{n}+p_{n-1} X_{n}, \\
r_{n} X_{n}=-Z_{n+1}+s_{n} Y_{n+1}+p_{n} X_{n+1}, \\
r_{n} Y_{n}=-Y_{n+2}+s_{n} X_{n+2}-q_{n} X_{n+1}, \\
r_{n} Z_{n}=-X_{n+3}-p_{n} X_{n+2}-q_{n} Y_{n+1}, \\
r_{n+1} Z_{n+1}=-X_{n+4}-p_{n+1} X_{n+3}-q_{n+1} Y_{n+2} .
\end{array}\right.
$$


We can solve this system by expressing the unknowns $Y_{n}, Y_{n+1}, Y_{n+2}, Z_{n+1}, Z_{n+2}$ in terms of $X_{n-1}, X_{n}, X_{n+1}, X_{n+2}, X_{n+3}$ and $X_{n+4}$. In particular, we obtain an expression for $Y_{n}$ in terms of $X_{n-1}, \ldots, X_{n+4}$ and another for $Y_{n+1}$ in terms of $X_{n-1}, \ldots, X_{n+4}$. In the first expression, we change $n$ to $n+1$ and equate the two expressions obtained for $Y_{n+1}$. This yields a relation between $X_{n-1}, \ldots, X_{n+5}$, which is (5.5) (with $n$ changed to $n+1$ ).

5.2. Orthogonal polynomials and recurrences. Let $L$ denote any path (closed or not) in $\mathbb{C}$ and a weight function $\omega$ defined on $L$ such that $\int_{L}\left|t^{k} \omega(t)\right||\mathrm{d} t|<+\infty$ for any integer $k \geq 0$. It follows that the integral $\mathcal{F}(z)=\int_{L} \frac{\omega(t)}{z-t} \mathrm{~d} t$ is convergent for any $z \notin L$. Let us consider a sequence of polynomials $\mathcal{A}_{n}(z) \in \mathbb{C}[z]$ such that

$$
\int_{L} t^{k} \mathcal{A}_{n}(t) \omega(t) \mathrm{d} t=0
$$

for any $k \in\left\{0,1, \ldots, \lambda_{n}\right\}$ where $\left(\lambda_{n}\right)_{n \geq 0}$ is a strictly increasing sequence. Finally, let us define

$$
\mathcal{R}_{n}(z)=\int_{L} \mathcal{A}_{n}(t) \frac{\omega(t)}{z-t} \mathrm{~d} t
$$

which is convergent for any $n \geq 0$ and any $z \notin L$.

Proposition 7. (i) Let us assume that $\left(\mathcal{A}_{n}(z)\right)_{n \geq 0}$ satisfies a recurrence

$$
\sum_{k=0}^{d} p_{k, n}(z) U_{n+k}=0,
$$

where $p_{k, n}(z)$ is a polynomial in $\mathbb{C}[z]$ of degree bounded by $\alpha_{k}$ independently of $n$. Then, $\left(\mathcal{R}_{n}(z)\right)_{n \geq N}$ is also a solution of (5.7), where $N=\min \left\{n \geq 0: \lambda_{n+k} \geq\right.$ $\left.\alpha_{k}, k=0, \ldots, d\right\}$ is independent of $z \notin L$.

(ii) Conversely, let us assume that $\left(\mathcal{R}_{n}(z)\right)_{n \geq 0}$ satisfies a recurrence

$$
\sum_{k=0}^{d} p_{k, n}(z) U_{n+k}=0,
$$

where $p_{k, n}(z)$ is a polynomial in $\mathbb{C}[z]$ of degree bounded by $\alpha_{k}$ independently of $n$. Let us also assume that $\mathcal{F}(z) \notin \mathbb{C}(z)$. Then, $\left(\mathcal{A}_{n}(z)\right)_{n \geq N}$ is also a solution of (5.8), where $N$ has the same definition as in $(i)$.

Remark 5. When $\left(\mathcal{A}_{n}(z)\right)_{n \geq 0}$ is a hypergeometric sequence in the sense defined by Wilf and Zeilberger [20], then the theory developed by these authors shows that $\left(\mathcal{A}_{n}(z)\right)_{n \geq 0}$ satisfies a linear recurrence of finite order. It is possible to bound a priori the order but it is usually a very crude bound and computing the coefficients of the recurrence is often impossible by hand. For any given hypergeometric example, Zeilberger's computer programs 22. enable us to compute the recurrence of lowest order (at least in principle). This is what we will do in the following sections.

Proof. (i) Since $\left(\mathcal{A}_{n}(z)\right)_{n \geq 0}$ satisfies (5.7), we have

$$
\int_{L}\left(\sum_{k=0}^{d} p_{k, n}(t) \mathcal{A}_{n+k}(t)\right) \frac{\omega(t)}{z-t} \mathrm{~d} t=0
$$


for any $z \notin L$ and any $n \geq 0$. Written differently, this means that

$$
\sum_{k=0}^{d} p_{k, n}(z) \mathcal{R}_{n+k}(z)=\sum_{k=0}^{d} \int_{L} \frac{p_{k, n}(t)-p_{k, n}(z)}{t-z} \mathcal{A}_{n+k}(t) \omega(t) \mathrm{d} t .
$$

We now remark that, for any $k=0, \ldots, d$, the function $\frac{p_{k, n}(t)-p_{k, n}(z)}{t-z}$ is a polynomial in $t$ of degree bounded by $\alpha_{k}-1$, independently of $n$. Therefore, by the orthogonality (5.6), we have

$$
\int_{L} \frac{p_{k, n}(t)-p_{k, n}(z)}{t-z} \mathcal{A}_{n+k}(t) \omega(t) \mathrm{d} t=0
$$

for $n$ such that $\lambda_{n+k} \geq \alpha_{k}$, in particular for $n \geq N$ because $\left(\lambda_{n}\right)_{n \geq 0}$ is strictly increasing. Hence, the right hand side of (5.9) is 0 and the assertion $(i)$ follows.

(ii) Let $K_{n}(z)=\sum_{k=0}^{d} p_{k, n}(z) \mathcal{A}_{n+k}(z)$, which we want to prove is 0 . Using that $\sum_{k=0}^{d} p_{k, n}(z) \mathcal{R}_{n+k}(z)=0$, we see that

$$
\int_{L} \frac{K_{n}(t)}{z-t} \omega(t) \mathrm{d} t=\sum_{k=0}^{d} \int_{L} \frac{p_{k, n}(z)-p_{k, n}(t)}{t-z} \mathcal{A}_{n+k}(t) \omega(t) \mathrm{d} t .
$$

We are in the same situation as above: each integral is 0 for $n$ such that $\lambda_{n+k} \geq \alpha_{k}$, in particular for $n \geq N$. Hence $\int_{L} \frac{K_{n}(t)}{z-t} \omega(t) \mathrm{d} t=0$ for any $z \notin L$ provided $n$ is large enough: we can rewrite this equation as

$$
\int_{L} \frac{K_{n}(z)-K_{n}(t)}{z-t} \omega(t) \mathrm{d} t=K_{n}(z) \mathcal{F}(z) .
$$

We now observe that the left hand side belongs to $\mathbb{C}[z]$ while the right hand side does not belong to $\mathbb{C}(z)$ if $K_{n}(z)$ is not identically 0 , by the irrationality hypothesis made on $\mathcal{F}$. Thus, we necessarily have $K_{n}(z)=0$ for $n \geq N$.

5.3. Birkhoff-Trjitzinsky theory. Many sequences in mathematics satisfy a linear recurrence of finite order. It is often an important problem to bound the growth of the sequence and this can be done using Birkhoff-Trjitzinsky theory [7] of linear recurrence of finite order, which encompasses the widely used Poincaré-Perron theory and which we now briefly describe 4

Consider a linear recurrence of order $\sigma$ of the form

$$
\sum_{k=0}^{\sigma} p_{k}(n) U_{n+k}=0,
$$

with $p_{0}(n)=1$ and $p_{\sigma}(n) \neq 0$ for all $n \geq 0$. These conditions ensure that, given any arbitrary initial values $U_{0}, U_{1}, \ldots, U_{\sigma-1}$, there exists a unique solution $\left(U_{n}\right)_{n \geq 0}$ to (5.10). We also assume that the coefficients $p_{k}(n)$ of (5.10) all admit an asymptotic expansion of Poincaré type as $n \rightarrow+\infty$ :

$$
p_{k}(n) \sim n^{e_{k} / \omega} \sum_{j=0}^{\infty} c_{j, k} n^{-j / k},
$$

where $e_{k} \in \mathbb{Z}, \omega \geq 1$ is an integer independent of $k$ and $c_{0, k} \neq 0$ unless $p_{k}(n) \equiv 0$.

The very difficult question addressed by Birkhoff and Trjitzinsky was to find a description of all possible asymptotic behaviors of the solutions of (5.10) as $n \rightarrow$

\footnotetext{
${ }^{4}$ This presentation is borrowed from 21].
} 
$+\infty$. For this, they introduced the following special class of asymptotic expansions. Consider a formal expansion $F(n)$ of the form

$$
F(n)=n^{\mu_{0} n} \exp \left(\sum_{k=1}^{\rho} \mu_{k} n^{(\rho+1-k) / \rho}\right) n^{\theta} \sum_{j=0}^{t} \ln (n)^{j} n^{r_{t-j / \rho}} q_{j}(\rho ; n),
$$

where $q_{j}(\rho ; n)=\sum_{s=0}^{\infty} b_{s, j} n^{-s / \rho}, \rho, r_{j}, \mu_{0} \rho$ are integers such that $\rho \geq 1, r_{0}=0$, $\mu_{j}, \theta, b_{s, j}$ are complex numbers, $-\pi \leq \operatorname{Im}\left(\mu_{1}\right)<\pi$ and $b_{0, j} \neq 0$ unless $b_{s, j}=0$ for all $s \geq 0$.

An expansion $F(n)$ is called a formal solution of (5.10) if, when it is substituted in (5.10), the coefficients of each quantity $n^{\theta+r / \rho+s / \omega} \ln (n)^{j}, j=0,1, \ldots, t, r, s=$ $0, \pm 1, \pm 2, \ldots$, are all 0 .

Proposition 8 (Birkhoff-Trjitzinsky). (i) There exist $\sigma$ formal solutions $\left(F_{j}(n)\right)_{j=1, \ldots, \sigma}$ of (5.10), unique up to a constant multiple, with $\omega$ dividing $\rho_{j}$.

(ii) Each such formal solution $F_{j}(n)$ describes the asymptotic behavior of a particular solution $\left(U_{j, n}\right)_{n \geq 0}$ of (5.10).

(iii) These special solutions $\left(U_{j, n}\right)_{n \geq 0}, j=1, \ldots, \sigma$, form a basis over $\mathbb{C}$ of the space of solutions of (5.10).

In practice, given a recurrence of the form (5.10), one first tries to construct a formal solution $F(n)$ without logarithmic terms and with $q_{0}(\rho ; n)$ reduced to its first term; i.e., $F(n)$ is of the form

$$
F(n)=n^{\mu_{0} n} \exp \left(\mu_{1} n\right) \exp \left(\alpha_{1} n^{\beta}+\alpha_{2} n^{\beta-1 / \rho}+\alpha_{3} n^{\beta-2 / \rho}+\cdots\right) n^{\theta},
$$

where $\alpha_{1} \neq 0, \beta=m / \rho$ for some $0<m<\rho$. Then,

$$
\begin{aligned}
\frac{F(n+k)}{F(n)}=n^{\mu_{0} k} e^{\left(\mu_{0}+\mu_{1}\right) k}\left(1+\frac{k \theta+k^{2} \mu_{0} / 2}{n}+\cdots\right) & \\
& \times \exp \left(\alpha_{1} \beta k n^{\beta-1}+\alpha_{2}(\beta-1 / \rho) k n^{\beta-1 / \rho-1}+\cdots\right) .
\end{aligned}
$$

Assuming that $F(n)$ formally satisfies the recurrence (5.10), we have that $\sum_{k=0}^{\sigma} p_{k}(n) F(n+k) / F(n)=0$. The formal substitution in this equation of the asymptotic expansions (5.11) and (5.13) of $p_{k}(n)$ and $F(n+k) / F(n)$ respectively should then hopefully yield enough equations in the unknown data to obtain $\sigma$ formal solutions. If this simplified approach fails (i.e., we do not get $\sigma$ different formal solutions), then we have to introduce logarithmic terms in $F(n) 5$ Many examples obtained using the above strategy are described in [21].

\section{Study of the Padé approximants in the Case $s=2$}

We now study in detail the case $s=2$ of the previous sections. Our goal is to find explicit expressions and the precise asymptotic behavior of the polynomials and remainder functions that constitute the Padé approximants of type II.

6.1. Explicit expressions for the polynomials. To simplify the notation, we denote by $A_{n}(z)$ the Laguerre-type polynomial $A_{2, n}(z)$. Explicitly, we have

$$
A_{n}(z)=\frac{1}{n !^{2}} e^{z}\left(z^{n}\left(z^{n} e^{-z}\right)^{(n)}\right)^{(n)}, n \geq 0 .
$$

\footnotetext{
${ }^{5}$ In our case, this procedure will work fine without logarithmic terms.
} 
The polynomials $B_{1,2, n}(z)$ and $B_{2,2, n}(z)$ become respectively

$$
B_{n}(z)=\int_{0}^{\infty} \frac{A_{n}(t)-A_{n}(z)}{z-t} e^{-t} \mathrm{~d} t
$$

and

$$
C_{n}(z)=\int_{0}^{\infty} \frac{A_{n}(t)-A_{n}(z)}{z-t} \ln (t) e^{-t} \mathrm{~d} t,
$$

and the remainder functions $R_{1,2, n}(z)$ and $R_{2,2, n}(z)$ become

$$
R_{1, n}(z)=A_{n}(z) \mathcal{E}_{1}(z)+B_{n}(z)=\int_{0}^{\infty} \frac{A_{n}(t)}{z-t} e^{-t} \mathrm{~d} t
$$

and

$$
R_{2, n}(z)=A_{n}(z) \mathcal{E}_{2}(z)+C_{n}(z)=\int_{0}^{\infty} \frac{A_{n}(t)}{z-t} \ln (t) e^{-t} \mathrm{~d} t .
$$

We now provide some explicit expressions for $A_{n}, B_{n}$ and $C_{n}$.

Proposition 9. Let

$$
a_{k, n}=\frac{(-1)^{k}}{k !} \sum_{j=0}^{k}\left(\begin{array}{c}
n \\
j
\end{array}\right)\left(\begin{array}{c}
n \\
k-j
\end{array}\right)\left(\begin{array}{c}
n+j \\
n
\end{array}\right) .
$$

Then we have

$$
\begin{aligned}
& A_{n}(z)=\sum_{k=0}^{2 n} a_{k, n} z^{k} \in \mathbb{Q}[z], \quad B_{n}(z)=-\sum_{k=0}^{2 n} a_{k, n} \sum_{j=0}^{k-1} j ! z^{k-j-1} \in \mathbb{Q}[z], \\
& C_{n}(z)=-\sum_{k=0}^{2 n} a_{k, n} \sum_{j=0}^{k-1} j !\left(H_{j}-\gamma\right) z^{k-j-1} \in \mathbb{Q}[z]+\gamma \mathbb{Q}[z] .
\end{aligned}
$$

The degrees of $A_{n}, B_{n}$ and $C_{n}$ are $2 n, 2 n-1$ and $2 n-1$, respectively.

Remarks 6. a) We note that $C_{n}(z)=-\gamma B_{n}(z)-D_{n}(z)$, where

$$
D_{n}(z)=\sum_{k=0}^{2 n} a_{k, n} \sum_{j=0}^{k-1} j ! H_{j} z^{k-j-1}
$$

has rational coefficients, as predicted by (4.2).

b) We also have $a_{k, n}=\frac{1}{k !} \sum_{j=0}^{k}(-1)^{j}\left(\begin{array}{c}k \\ j\end{array}\right)\left(\begin{array}{c}n+j \\ j\end{array}\right)^{2}$. Both expressions for $a_{k, n}$ are in fact ${ }_{3} F_{2}$-hypergeometric terminating series whose equality is a consequence of Thomae's 120 classical relations. (For a quick check, use the simple Maple code given in [14, p. 192].)

Proof. The expression for $A_{n}(z)$ is an immediate consequence of the definition (6.1) by the Leibniz formula. Then

$$
\begin{aligned}
B_{n}(z) & =-\sum_{k=0}^{2 n} a_{k, n} \int_{0}^{\infty} \frac{t^{k}-z^{k}}{t-z} e^{-t} \mathrm{~d} t=-\sum_{k=0}^{2 n} a_{k, n} \sum_{j=0}^{k-1} z^{k-j-1} \int_{0}^{\infty} t^{j} e^{-t} \mathrm{~d} t \\
& =-\sum_{k=0}^{2 n} a_{k, n} \sum_{j=0}^{k-1} j ! z^{k-j-1} .
\end{aligned}
$$


Similarly,

$$
\begin{aligned}
C_{n}(z) & =-\sum_{k=0}^{2 n} a_{k, n} \int_{0}^{\infty} \frac{t^{k}-z^{k}}{t-z} \ln (t) e^{-t} \mathrm{~d} t \\
& =-\sum_{k=0}^{2 n} a_{k, n} \sum_{j=0}^{k-1} z^{k-j-1} \int_{0}^{\infty} t^{j} \ln (t) e^{-t} \mathrm{~d} t \\
& =-\sum_{k=0}^{2 n} a_{k, n} \sum_{j=0}^{k-1} j !\left(H_{j}-\gamma\right) z^{k-j-1} .
\end{aligned}
$$

Finally, we note that $a_{2 n, n}$ is clearly not 0 and it is the coefficient of highest degree $2 n$ for $A_{n}$ and $-B_{n}$ (degree $\left.=2 n-1\right)$. Also, $(\gamma-1) a_{2 n, n} \neq 0$ is the coefficient of highest degree $(=2 n-1)$ of $C_{n}$.

6.2. Linear recurrence in the case $s=2$. Any sequence of multiple orthogonal polynomials of hypergeometric origin (and related objects) satisfies a linear recurrence of finite order with polynomial coefficients (in $n$ and $z$ ). This was proved by Wilf-Zeilberger in a general setting [20. However, it can be very difficult to explicitly compute such a recurrence without a computer.

Proposition 10. The six sequences $\left(A_{n}(z)\right)_{n \geq 0}, \quad\left(B_{n}(z)\right)_{n \geq 0}, \quad\left(C_{n}(z)\right)_{n \geq 0}$, $\left(D_{n}(z)\right)_{n \geq 0},\left(R_{1, n}(z)\right)_{n \geq 0}$ and $\left(R_{2, n}(z)\right)_{n \geq 0}$ are solutions of the third order linear recurrence

$$
\begin{aligned}
(n+3)^{2}(3 z-8 n-14) U_{n+3}= & -\left(24 n^{3}-57 z n^{2}+162 n^{2}+356 n+26 z^{2} n-258 z n-3 z^{3}\right. \\
\left.-285 z+59 z^{2}+254\right) U_{n+2}+ & \left(24 n^{3}+7 z n^{2}+138 n^{2}-6 z^{2} n+252 n+42 z n+47 z\right. \\
- & \left.9 z^{2}+150\right) U_{n+1}+(n+1)^{2}(3 z-8 n-22) U_{n} .
\end{aligned}
$$

(We must assume that $z \notin[0, \infty)$ in the case of $\left(R_{1, n}(z)\right)_{n \geq 0}$ and $\left(R_{2, n}(z)\right)_{n \geq 0}$.)

Proof. We will prove first that $\left(A_{n}(z)\right)_{n>0}$ satisfies (6.2). These polynomials correspond to the case $\alpha_{1}=\alpha_{2}=0, \beta=-1, n_{1}=n_{2}=n$ of the sequence of polynomials considered in [5, Sec. 3.2]. It would therefore probably be possible to deduce the recurrence for $A_{n}(z)$ from the pseudo-recurrence given there. Instead, we propose the following "computer assisted" proof.

By Cauchy's formula applied twice to the definition (6.1) of $A_{n}(z)$, we have

$$
A_{n}(z)=\frac{1}{(2 i \pi)^{2}} \int_{\mathscr{C}_{0} \times \widetilde{\mathscr{C}}_{0}} \frac{(u+z)^{n}(u+v+z)^{n}}{u^{n+1} v^{n+1}} e^{-(u+v)} \mathrm{d} u \mathrm{~d} v,
$$

where $\mathscr{C}_{0}$ and $\widetilde{\mathscr{C}}_{0}$ denote circles of center 0 and arbitrary radii. The continuous version of Zeilberger's algorithm 6 then shows automatically that the function

$$
F_{n}(u, v)=\frac{(u+z)^{n}(u+v+z)^{n}}{u^{n+1} v^{n+1}} e^{-(u+v)}
$$

\footnotetext{
${ }^{6}$ We used the version MultInt implemented in Maple by A. Tefara [8]. It took less than 7 seconds to get (6.3).
} 
satisfies the identity

$$
\begin{aligned}
& (6.3) \quad-(n+3)^{2}(3 z-8 n-14) F_{n+3}(u, v) \\
& -\left(24 n^{3}-57 z n^{2}+162 n^{2}+356 n+26 z^{2} n-258 z n-3 z^{3}-285 z+59 z^{2}+254\right) F_{n+2}(u, v) \\
& \quad+\left(24 n^{3}+7 z n^{2}+138 n^{2}-6 z^{2} n+252 n+42 z n+47 z-9 z^{2}+150\right) F_{n+1}(u, v) \\
& +(n+1)^{2}(3 z-8 n-22) F_{n}(u, v)=\frac{\partial}{\partial u}\left(\frac{P_{n}(u, v) F_{n}(u, v)}{u^{2} v^{3}}\right)+\frac{\partial}{\partial v}\left(\frac{Q_{n}(u, v) F_{n}(u, v)}{u^{3} v^{2}}\right),
\end{aligned}
$$

where $P_{n}(u, v)$ and $Q_{n}(u, v)$ are some complicated but explicit polynomials in the variables $u, v, z$. Since

$$
\begin{aligned}
& \frac{1}{(2 i \pi)^{2}} \int_{\mathscr{C}_{0} \times \widetilde{\mathscr{C}}_{0}} \frac{\partial}{\partial u}\left(\frac{P_{n}(u, v) F_{n}(u, v)}{u^{2} v^{3}}\right) \mathrm{d} u \mathrm{~d} v=0, \\
& \frac{1}{(2 i \pi)^{2}} \int_{\mathscr{C}_{0} \times \widetilde{\mathscr{C}}_{0}} \frac{\partial}{\partial v}\left(\frac{Q_{n}(u, v) F_{n}(u, v)}{u^{3} v^{2}}\right) \mathrm{d} u \mathrm{~d} v=0,
\end{aligned}
$$

by integrating (6.3) over $\mathscr{C}_{0} \times \widetilde{\mathscr{C}}_{0}$, we see that the sequence $\left(A_{n}(z)\right)_{n \geq 0}$ is a solution of the recurrence (6.2).

To prove that $\left(R_{1, n}(z)\right)_{n \geq 0}$ and $\left(R_{2, n}(z)\right)_{n \geq 0}$ satisfy (6.2), at least for $n$ large enough, we apply Proposition (7), (i). The remaining values of $n$ are checked by direct computation. By linearity, we see that $\left(B_{n}(z)\right)_{n \geq 0},\left(C_{n}(z)\right)_{n \geq 0}$ and $\left(D_{n}(z)\right)_{n \geq 0}$ are also solutions of (6.2). This finishes the proof of the proposition.

6.3. Growth of the polynomial solutions of recurrence (6.2). Using the algorithmic method developed at the end of Section [5.3, it is a simple task to find three formal solutions of (6.2), which turn out to be without logarithmic terms. We omit the details, which are purely computational.

Proposition 11. For any $z \in \mathbb{C}^{\star}$, the recurrence (6.2) admits three formal solutions whose expansions as $n \rightarrow+\infty$ are:

$$
\begin{gathered}
f_{1}(n, z)=\exp \left(-3 z^{1 / 3} n^{2 / 3}+z^{2 / 3} n^{1 / 3}\right)\left(\frac{1}{n}+\mathcal{O}\left(\frac{1}{n^{2}}\right)\right) \\
f_{2}(n, z)=\exp \left(3 e^{i \pi / 3} z^{1 / 3} n^{2 / 3}+e^{2 i \pi / 3} z^{2 / 3} n^{1 / 3}\right)\left(\frac{1}{n}+\mathcal{O}\left(\frac{1}{n^{2}}\right)\right), \\
f_{3}(n, z)=\exp \left(3 e^{-i \pi / 3} z^{1 / 3} n^{2 / 3}+e^{-2 i \pi / 3} z^{2 / 3} n^{1 / 3}\right)\left(\frac{1}{n}+\mathcal{O}\left(\frac{1}{n^{2}}\right)\right) .
\end{gathered}
$$

Remarks 7. a) If $z=0$, then the behavior of the solutions of (6.2) is different. We discard this case.

b) The leading coefficient of (6.2) written in the form (5.10) is

$$
-\frac{(n+3)^{2}(3 z-8 n-14)}{(n+1)^{2}(3 z-8 n-22)}
$$

The Birkhoff-Trjitzinsky theory applies provided this coefficient is well-defined and never 0, i.e., $z \notin \frac{14}{3}+\frac{8}{3} \mathbb{N}$. However, for $z \in \frac{14}{3}+\frac{8}{3} \mathbb{N}$, this theory still applies provided the recurrence (6.2) starts not at $n=0$ but at $n \gg_{z} 1$ (so that the leading coefficient is not 0 ). With this interpretation, Proposition 11 holds for any $z \in \mathbb{C}^{\star}$.

Note that for $x>0$, our choice of the branch of the logarithm ensures that $x^{1 / 3}$ is real and thus $>0$. 
Proposition 12. For any fixed real number $x>0$, there exists a constant $a(x) \geq 0$ such that

$$
\left|A_{n}(x)\right| \leq \frac{a(x)}{n} \exp \left(3 / 2 x^{1 / 3} n^{2 / 3}-1 / 2 x^{2 / 3} n^{1 / 3}\right)
$$

as $n \rightarrow+\infty$, and a similar result holds for $B_{n}(x), C_{n}(x), D_{n}(x)$, with a suitable constant for each.

Proof. Let $U_{n}$ denote any one of $A_{n}, B_{n}, C_{n}$ or $D_{n}$. Since (by Proposition 101) $\left(U_{n}(x)\right)_{n \geq 0}$ is a solution of (6.2), then by Propositions 8 and 11, there exists a real number $u(x) \geq 0$ such that $\left|U_{n}(x)\right| \leq u(x) \max \left(\left|f_{1}(n, x)\right|,\left|f_{2}(n, x)\right|,\left|f_{3}(n, x)\right|\right)$ as $n \rightarrow+\infty$. For $x>0$, the first exponential $f_{1}$ tends to 0 whereas $f_{2}, f_{3}$ are both bounded in modulus by $n^{-1} \exp \left(\frac{3}{2} x^{1 / 3} n^{2 / 3}-\frac{1}{2} x^{2 / 3} n^{1 / 3}\right)$. The result follows.

6.4. Growth of the remainder solutions of recurrence (6.2). From Proposition [11, we know that for any complex number $z \notin[0,+\infty)$ we can bound the growth of $R_{1, n}(z)$ and $R_{2, n}(z)$. However, we are not interested in these two functions separetely but rather in their linear combination $R_{n}(z)$ defined by

$$
R_{n}(z)=\log (z) R_{1, n}(z)-R_{2, n}(z)=\int_{0}^{\infty} A_{n}(t) \frac{\ln (t)-\log (z)}{t-z} e^{-t} \mathrm{~d} t .
$$

The choice of the principle branch for the logarithm ensures that $R_{n}$ is a holomorphic function in $\mathbb{C} \backslash(-\infty, 0]$, whereas $R_{1, n}$ and $R_{2, n}$ are holomorphic functions in $\mathbb{C} \backslash[0,+\infty)$.

Proposition 13. For any fixed real number $x>0$, there exists a constant $r(x) \neq 0$ such that

$$
R_{n}(x) \sim \frac{r(x)}{n} \exp \left(-3 x^{1 / 3} n^{2 / 3}+x^{2 / 3} n^{1 / 3}\right)
$$

as $n \rightarrow+\infty$.

Proof. We know that there exist some constants $r_{1}(x), r_{2}(x), r_{3}(x)$ such that

$$
R_{n}(x) \sim r_{1}(x) f_{1}(n, x)+r_{2}(x) f_{2}(n, x)+r_{3}(x) f_{3}(n, x)
$$

as $n \rightarrow+\infty$, where the equivalence must be understood in the sense that $R_{n}(x)=$ $r_{1}(x) u_{1}(n, x)+r_{2}(x) u_{2}(n, x)+r_{3}(x) u_{3}(n, x)$, where, for $j=1,2,3, u_{j}(n, x)$ is a special solution of the recurrence which has $f_{j}(n, x)$ as an asymptotic expansion. If $r_{2}(x)= \pm r_{3}(x)$, then a difficulty arises. Indeed, although $f_{2}(n, x)$ and $f_{3}(n, x)$ both tend to infinity with $n$, it is not immediate that this is also the case of $f_{2}(n, x) \pm$ $f_{3}(n, x)$ : a sine or a cosine enters the game, giving a quantity that can be small (making it difficult to see which term is preponderant).

Since $R_{n}(x)$ is real for $x>0$, we necessarily have $r_{2}(x)=r_{3}(x)$ and we must solve this difficulty. Using the expressions in Proposition [11, we have

$$
\begin{aligned}
& \text { (6.5) } f_{2}(n, x)+f_{3}(n, x) \\
& =\frac{2}{n} \exp \left(3 / 2 x^{1 / 3} n^{2 / 3}-1 / 2 x^{2 / 3} n^{1 / 3}\right)\left(\cos \frac{\sqrt{3}}{2}\left(3 x^{1 / 3} n^{2 / 3}+x^{2 / 3} n^{1 / 3}\right)+\mathcal{O}\left(\frac{1}{n}\right)\right) .
\end{aligned}
$$

We want to show that

$$
\limsup _{n \rightarrow+\infty}\left|f_{2}(n, x)+f_{3}(n, x)\right|=+\infty .
$$


In view of (6.5), it is enough to find infinitely many $n$ such that

$$
\left|\cos \frac{\sqrt{3}}{2}\left(3 x^{1 / 3} n^{2 / 3}+x^{2 / 3} n^{1 / 3}\right)\right| \geq 1 / 2,
$$

for example. To simplify, let $\alpha=x^{1 / 3}>0$ and $n=m^{3}$, with $m \in \mathbb{N}$. A classical theorem of Weyl says that for any polynomial $P(X) \in \mathbb{R}[X]$ with at least one irrational coefficient other than the constant, the sequence $(P(n))_{n \geq 0}$ is uniformly distributed mod 1. This theorem can be applied to $P(X)=\frac{\sqrt{3}}{4 \pi}\left(3 \alpha X^{2}+\alpha^{2} X\right)$ : indeed, if $\sqrt{3} \alpha / \pi \in \mathbb{Q}^{\star}$, then $\sqrt{3} \alpha^{2} / \pi \notin \mathbb{Q}$. Since $\cos \frac{\sqrt{3}}{2}\left(3 \alpha m^{2}+\alpha^{2} m\right)=\cos (2 \pi\{P(m)\})$, the equidistribution of $\{P(m)\}$ in [0,1] implies the density of $\cos \frac{\sqrt{3}}{2}\left(3 \alpha m^{2}+\alpha^{2} m\right)$ in $[-1,1]$. This is more than what is needed to prove that (6.7) holds for infinitely many $n$. Since along the subsequence of integers for which (6.7) holds, $f_{2}(n, x)$ and $f_{3}(n, x)$ are $\mathcal{O}\left(\left|f_{2}(n, x)+f_{3}(n, x)\right|\right)$, we obtain that

$$
\limsup _{n \rightarrow+\infty}\left|u_{2}(n, x)+u_{3}(n, x)\right|=+\infty .
$$

From (6.8), we now deduce that the property " $r_{2}(x)=r_{3}(x)=0$ " is a consequence of " $R_{n}(x) \rightarrow 0$ as $n \rightarrow+\infty$ ". To show this fact, we first prove the following alternative expression for $R_{n}(x)$, which is interesting in itself.

Lemma 1. For any $x>0$, we have

$$
R_{n}(x)=\int_{0}^{\infty} \int_{0}^{\infty} \frac{t^{n} v^{n} e^{-t}}{(1+v)^{n+1}(x+v t)^{n+1}} \mathrm{~d} t \mathrm{~d} v .
$$

Remark 8. By analytic continuation, (6.9) holds for any $x \in \mathbb{C} \backslash(-\infty, 0]$.

To prove Lemma 1, we use the easily proved identity

$$
\frac{\log (t)-\log (x)}{t-x}=\int_{0}^{\infty} \frac{\mathrm{d} u}{(1+u)(x+u t)}
$$

that holds for any $t>0$, any $x \in \mathbb{C} \backslash(-\infty, 0]$ and with the principal branch of the $\log$. Let $L_{n}(t)=e^{t}\left(t^{n} e^{-t}\right)^{(n)} / n$ !. We have $A_{n}(t)=e^{t}\left(t^{n} e^{-t} L_{n}(t)\right)^{(n)} / n$ ! and thus

$$
R_{n}(x)=\int_{0}^{\infty} \int_{0}^{\infty} \frac{A_{n}(t) e^{-t}}{(1+u)(x+u t)} \mathrm{d} t \mathrm{~d} u=\int_{0}^{\infty} \int_{0}^{\infty} \frac{u^{n} t^{n} L_{n}(t) e^{-t}}{(1+u)(x+u t)^{n+1}} \mathrm{~d} t \mathrm{~d} u,
$$

where we have integrated $n$ times by parts with respect to $t$ and where the interchange of integrals is justified by Fubini's theorem. We now make the change of variable $u \rightarrow v$ defined by $u=x /(t v)$ and obtain that

$$
R_{n}(x)=\int_{0}^{\infty} \int_{0}^{\infty} \frac{L_{n}(t) e^{-t}}{(1+v)^{n+1}(x+v t)} \mathrm{d} t \mathrm{~d} v=\int_{0}^{\infty} \int_{0}^{\infty} \frac{v^{n} t^{n} e^{-t}}{(1+v)^{n+1}(x+v t)^{n+1}} \mathrm{~d} t \mathrm{~d} v
$$

where we have again integrated $n$ times by parts with respect to $t$. This is exactly (6.9).

We are now ready to prove that $R_{n}(x) \rightarrow 0$ as $n \rightarrow+\infty$ for any $x>0$. Indeed, in this case, we trivially have $0 \leq \frac{v^{n} t^{n}}{(x+v t)^{n+1}} \leq \frac{1}{x}$ for $u, v \geq 0$ and thus (for $n \geq 1$ to ensure convergence) we have

$$
\left|R_{n}(x)\right| \leq \frac{1}{x} \int_{0}^{\infty} \int_{0}^{\infty} \frac{e^{-t}}{(1+v)^{n+1}} \mathrm{~d} t \mathrm{~d} v=\frac{1}{x n} .
$$

It remains to prove that $r_{1}(x) \neq 0$ : if it were equal to 0 , we would have $R_{n}(x)=0$ for any $n \geq 0$. This is not the case because the integral representation (6.9) of 
$R_{n}(x)$ shows that $R_{n}(x)>0$ for all $n \geq 0$ and $x>0$. This finishes the proof of Proposition 13 .

Remark 9. The integral (6.9) can be used to bound precisely $R_{n}(x)$.

7. Construction of rational approximations to $\ln (x)+\gamma$

For simplicity, set $\mathcal{E}(z)=\log (z) \mathcal{E}_{1}(z)-\mathcal{E}_{2}(z)$ and $\mathcal{G}(z)=\log (z)+\gamma$. We have shown that

$$
\begin{aligned}
& R_{1, n}(z)=A_{n}(z) \mathcal{E}_{1}(z)+B_{n}(z) \\
& R_{2, n}(z)=A_{n}(z) \mathcal{E}_{2}(z)-B_{n}(z) \gamma-D_{n}(z)
\end{aligned}
$$

Thus

$$
R_{n}(z)=\log (z) R_{1, n}(z)-R_{2, n}(z)=A_{n}(z) \mathcal{E}(z)+B_{n}(z) \mathcal{G}(z)+D_{n}(z) .
$$

7.1. Linear recurrence for $\ln (x)+\gamma$.

Proposition 14. We define the three determinants

$$
Q_{n}(z)=\left|\begin{array}{ll}
A_{n}(z) & B_{n}(z) \\
A_{n+1}(z) & B_{n+1}(z)
\end{array}\right| \in \mathbb{Q}[z], \quad P_{n}(z)=\left|\begin{array}{ll}
D_{n}(z) & A_{n}(z) \\
D_{n+1}(z) & A_{n+1}(z)
\end{array}\right| \in \mathbb{Q}[z]
$$

and

$$
S_{n}(z)=\left|\begin{array}{ll}
A_{n}(z) & R_{n}(z) \\
A_{n+1}(z) & R_{n+1}(z)
\end{array}\right| .
$$

(i) For any $x>0$, we have $S_{n}(x)=Q_{n}(x)(\ln (x)+\gamma)-P_{n}(x)$.

(ii) The sequences $\left(P_{n}(z)\right)_{n \geq 0},\left(Q_{n}(z)\right)_{n \geq 0},\left(S_{n}(z)\right)_{n \geq 0}$ all satisfy the recurrence

$$
\begin{aligned}
& \text { (7.1) } \quad(n+3)^{2}(n+4)^{2}(3 z-8 n-14)(3 z-8 n-22) U_{n+3} \\
& =-\left(24 n^{3}+7 z n^{2}+210 n^{2}-6 z^{2} n+56 z n+600 n+96 z-15 z^{2}+564\right)(n+3)^{2}(3 z-8 n-14) U_{n+2} \\
& +\left(24 n^{3}-57 z n^{2}+162 n^{2}+356 n+26 z^{2} n-258 z n-3 z^{3}-285 z+59 z^{2}+254\right) \\
& \cdot(n+2)^{2}(3 z-8 n-30) U_{n+1} \\
& +(n+1)^{2}(n+2)^{2}(3 z-8 n-22)(3 z-8 n-30) U_{n} .
\end{aligned}
$$

Proof. (i) We have

$$
\begin{aligned}
S_{n} & =\left|\begin{array}{ll}
A_{n} & R_{n} \\
A_{n+1} & R_{n+1}
\end{array}\right|=\left|\begin{array}{ll}
A_{n} & A_{n} \mathcal{E}+B_{n} \mathcal{G}+D_{n} \\
A_{n+1} & A_{n+1} \mathcal{E}+B_{n+1} \mathcal{G}+D_{n+1}
\end{array}\right| \\
& =\left|\begin{array}{ll}
A_{n} & A_{n} \\
A_{n+1} & A_{n+1}
\end{array}\right| \mathcal{E}+\left|\begin{array}{ll}
A_{n} & B_{n} \\
A_{n+1} & B_{n+1}
\end{array}\right| \mathcal{G}+\left|\begin{array}{ll}
A_{n} & D_{n} \\
A_{n+1} & D_{n+1}
\end{array}\right| \\
& =Q_{n} \mathcal{G}-P_{n}
\end{aligned}
$$

as desired.

(ii) We apply Proposition 5 in the case $d=2$, i.e., Eq. (5.3), to the recurrence (6.2). This works for $n$ large enough. The remaining values of $n$ are checked by a direct computation.

We have thus constructed rational "approximations" to $\ln (x)+\gamma$. Of course, we have yet to prove that they are really approximations and we will study their properties in the rest of this section. 
7.2. Asymptotic behavior of the solutions of recurrence (1.3). Using the Birkhoff-Trjitzinsky theory as we did in the previous section, it is not hard to analyse the recurrence (1.3). Again, we skip the details of the computations.

Proposition 15. For any $z \in \mathbb{C}^{\star}$, the recurrence (6.2) admits three formal solutions whose leading terms as $n \rightarrow+\infty$ are:

$$
\begin{gathered}
\widetilde{f}_{1}(n, z)=\exp \left(3 z^{1 / 3} n^{2 / 3}-z^{2 / 3} n^{1 / 3}\right)\left(\frac{1}{n^{2}}+\mathcal{O}\left(\frac{1}{n^{3}}\right)\right), \\
\widetilde{f}_{2}(n, z)=\exp \left(3 e^{2 i \pi / 3} z^{1 / 3} n^{2 / 3}-e^{-2 i \pi / 3} z^{2 / 3} n^{1 / 3}\right)\left(\frac{1}{n^{2}}+\mathcal{O}\left(\frac{1}{n^{3}}\right)\right), \\
\widetilde{f}_{3}(n, z)=\exp \left(3 e^{-2 i \pi / 3} z^{1 / 3} n^{2 / 3}-e^{2 i \pi / 3} z^{2 / 3} n^{1 / 3}\right)\left(\frac{1}{n^{2}}+\mathcal{O}\left(\frac{1}{n^{3}}\right)\right) .
\end{gathered}
$$

Our next task is to use this proposition to find the asymptotic behavior of the sequences $\left(P_{n}(x)\right)_{n \geq 0},\left(Q_{n}(x)\right)_{n \geq 0}$ and $\left(S_{n}(x)\right)_{n \geq 0}$ for $x>0$.

Proposition 16. For any real number $x>0$, there exists two constants $q(x) \neq 0$ and $s(x) \neq 0$ such that

$$
\left|S_{n}(x)\right| \leq \frac{s(x)}{n^{2}} \exp \left(-\frac{3}{2} x^{1 / 3} n^{2 / 3}+\frac{1}{2} x^{2 / 3} n^{1 / 3}\right)
$$

and

$$
Q_{n}(x) \sim \frac{q(x)}{n^{2}} \exp \left(3 x^{1 / 3} n^{2 / 3}-x^{2 / 3} n^{1 / 3}\right)
$$

as $n \rightarrow+\infty$. Furthermore, for any $x>0, S_{n}(x) \neq 0$ for infinitely many integers $n$.

If $x \neq e^{-\gamma}$, then $P_{n}(x) \sim(\ln (x)+\gamma) Q_{n}(x)$ as $n \rightarrow+\infty$. If $x=e^{-\gamma}$, then $P_{n}(x)=-S_{n}(x)$.

Proof. Let us fix a real number $x>0$. We define two other sequences:

$$
M_{n}(x)=\left|\begin{array}{ll}
B_{n}(x) & D_{n}(x) \\
B_{n+1}(x) & D_{n+1}(x)
\end{array}\right| \in \mathbb{Q}[x] \text { and } T_{n}(x)=\left|\begin{array}{ll}
R_{n}(x) & B_{n}(x) \\
R_{n+1}(x) & B_{n+1}(x)
\end{array}\right| .
$$

Both are solutions of the recurrence (6.2) and a computation similar to the one in the proof of Proposition 14 shows that $T_{n}(x)=Q_{n}(x) \mathcal{E}(x)-M_{n}(x)$.

We will first prove that $\left(Q_{n}(x)\right)_{n \geq 0},\left(P_{n}(x)\right)_{n \geq 0}$ and $\left(M_{n}(x)\right)_{n \geq 0}$ form a basis over $\mathbb{C}$ of the space of solutions of (6.2). It is enough to prove that, for any fixed $x$, if $n$ is large enough (with respect to $x$ ), then the determinant

$$
\Delta_{n}(x)=\left|\begin{array}{lll}
Q_{n}(x) & Q_{n+1}(x) & Q_{n+2}(x) \\
P_{n}(x) & P_{n+1}(x) & P_{n+2}(x) \\
M_{n}(x) & M_{n+1}(x) & M_{n+2}(x)
\end{array}\right|
$$

is not zero. Using the recurrence (1.3) in the last column of $\Delta_{n}(x)$, we find that, for any $n \geq 1$,

$$
\Delta_{n}(x)=\frac{n^{2}(n+1)^{2}(3 x-8 n-22)}{(n+2)^{2}(n+3)^{2}(3 x-8 n-6)} \Delta_{n-1}(x)
$$


and hence (after iteration and simplification)

$$
\begin{aligned}
\Delta_{n}(x) & =\Delta_{0}(x) \prod_{j=0}^{n-1} \frac{(n-j)^{2}(n-j+1)^{2}(3 x-8(n-j)-22)}{(n-j+2)^{2}(n-j+3)^{2}(3 x-8(n-j)-6)} \\
& =\Delta_{0}(x) \frac{144(3 x-8 n-22)(3 x-8 n-14)}{(3 x-22)(3 x-14)(n+1)^{2}(n+2)^{4}(n+3)^{2}} .
\end{aligned}
$$

The determinant $\Delta_{0}(x)$ is easily computed using, amongst others, $A_{0}(x)=D_{1}(x)=$ $1, B_{0}(x)=D_{0}(x)=0: \Delta_{0}(x)=\frac{1}{144}(3 x-14)(3 x-22)$. Finally, we obtain

$$
\Delta_{n}(x)=\frac{(3 x-8 n-22)(3 x-8 n-14)}{(n+1)^{2}(n+2)^{4}(n+3)^{2}},
$$

which is not equal to 0 for $n>(3 x-14) / 8$. (This expression is not a surprise because $\Delta_{n}(x)$ is a polynomial in $x$.)

We observe that, for $x>0$, Proposition 15] shows that the only formal solution of the recurrence (1.3) that tends to infinity is equivalent to $\widetilde{f}_{1}(n, x)$. Therefore, since the sequences $Q_{n}(x), P_{n}(x)$ and $M_{n}(x)$ are independent, at least one of them must be equivalent to $\widetilde{f}_{1}(n, x)$ (up to some non-zero constant), in accordance with the Birkhoff-Trjitzinsky theory recalled in Proposition 8 .

Let us assume that $Q_{n}(x)$ is not equivalent to $c_{0}(x) \widetilde{f}_{1}(n, x)$ : it must then tend to 0 as $n \rightarrow+\infty$. By Propositions 12 and 13 , the determinants $S_{n}(x)$ and $T_{n}(x)$ are trivially bounded in modulus by

$2 \frac{a(x)}{n+1} e^{\frac{3}{2} x^{1 / 3}(n+1)^{2 / 3}-\frac{1}{2} x^{2 / 3}(n+1)^{1 / 3}} \times \frac{|r(x)|}{n} e^{-3 x^{1 / 3} n^{2 / 3}+x^{2 / 3} n^{1 / 3}} \leq c_{1}(x) e^{-c_{2}(x) n^{2 / 3}}$,

for some constants $c_{1}(x)>0, c_{2}(x)>0$ and $n$ large enough; hence both determinants tend to 0 as $n \rightarrow+\infty$. Since $P_{n}(x)=Q_{n}(x)(\ln (x)+\gamma)-S_{n}(x)$ and $M_{n}(x)=Q_{n}(x) \mathcal{E}(x)-T_{n}(x)$, we deduce that $P_{n}(x)$ and $M_{n}(x)$ also both tend to 0 as $n \rightarrow+\infty$. However, this contradicts what we said in the previous paragraph. Thus the asymptotic behavior of $Q_{n}(x)$ is given by $\widetilde{f}_{1}(n, x)$.

If $x \neq e^{-\gamma}$, then $\ln (x)+\gamma \neq 0$ and therefore the equation $P_{n}(x)=Q_{n}(x)$ $(\ln (x)+\gamma)-S_{n}(x)$ shows that $P_{n}(x)$ behaves like $Q_{n}(x)$. However, if $x=e^{-\gamma}$, then $P_{n}(x)=-S_{n}(x)$.

Moreover, the function $(\ln (x)-\ln (t)) /(x-t)$ is positive for any $t, x>0$; thus $\mathcal{E}(x)>0$ for any $x>0$. It follows that $M_{n}(x) \sim Q_{n}(x) \mathcal{E}(x)$ as $n \rightarrow+\infty$.

It remains to find an asymptotic bound for $S_{n}(x)$. We know that there exist three constants $s_{1}(x), s_{2}(x), s_{3}(x)$ such that

$$
S_{n}(x) \sim s_{1}(x) \widetilde{f}_{1}(n, x)+s_{2}(x) \widetilde{f}_{2}(n, x)+s_{3}(x) \widetilde{f}_{3}(n, x),
$$

where one must be careful with the meaning of the equivalence symbol when $s_{2}(x)=$ $s_{3}(x)$ for the same reasons as those in the proof of Proposition 13. We proved above that $S_{n}(x) \rightarrow 0$; thus necessarily $s_{1}(x)=0$. Furthermore, $S_{n}(x)$ is real for $x$ real; thus $s_{2}(x)=s_{3}(x)$ and therefore we only obtain an upper bound:

$$
\begin{aligned}
S_{n}(x) \ll & \frac{\left|s_{2}(x)\right|}{n^{2}} \exp \left(-\frac{3}{2} x^{1 / 3} n^{2 / 3}+\frac{1}{2} x^{2 / 3} n^{1 / 3}\right) \\
& \times\left|\cos \frac{\sqrt{3}}{2}\left(3 x^{1 / 3} n^{2 / 3}+x^{2 / 3} n^{1 / 3}\right)+\mathcal{O}\left(\frac{1}{n}\right)\right| .
\end{aligned}
$$


Of course, this is more precise than the claim made in the statement of Proposition 16. The value of the cosine can be small because its image is dense in $[-1,1]$ as $n \rightarrow+\infty$ as shown during the proof of Proposition 13, where the same cosine occurred. It is therefore difficult to say something better than that.

Furthermore, we cannot have $S_{n}(x)=0$ for all $n \gg_{x} 1$. Indeed, since $S_{n}(x)=$ $Q_{n}(x)(\ln (x)+\gamma)-P_{n}(x)$ and $\ln (x)+\gamma$ is an irrational function, we would deduce that $Q_{n}(x)$ and $P_{n}(x)$ are identically 0 for $n \gg_{x} 1$ and therefore that $\Delta_{n}(x)=0$ for $n \gg_{x} 1$. But we have shown above that this not the case. Hence, in particular, $s_{2}(x) \neq 0$.

7.3. Coefficients and degrees of $P_{n}(z)$ and $Q_{n}(z)$. In this section, we prove the following result.

Proposition 17. The coefficients of the polynomials $(n+1) !^{2} P_{n}(z)$ and $(n+1) !^{2} Q_{n}(z)$ are integers and their degrees are at most $n+1$.

Proof. We define a sequence $v_{n}(z)=(n+1) !^{2} u_{n}(z)$, where $u_{n}$ denotes either $P_{n}$ or $Q_{n}$. The sequence $\left(v_{n}(z)\right)_{n \geq 0}$ satisfies the recurrence

$$
\begin{aligned}
& (3 z-8 n-14)(3 z-8 n-22) v_{n+3}(z) \\
& \quad=\alpha_{n}(z) v_{n+2}(z)+\beta_{n}(z)(n+2)^{2} v_{n+1}(z)+\delta_{n}(z)(n+1)^{2}(n+2)^{4} v_{n}(z),
\end{aligned}
$$

where $\delta_{n}(z)=(3 z-8 n-22)(3 z-8 n-30)$ and

$$
\begin{aligned}
\alpha_{n}(z)= & -\left(24 n^{3}+7 z n^{2}+210 n^{2}-6 z^{2} n+56 z n+600 n+96 z-15 z^{2}+564\right)(3 z-8 n-14), \\
\beta_{n}(z)= & \left(24 n^{3}-57 z n^{2}+162 n^{2}+356 n+26 z^{2} n\right. \\
& \left.-258 z n-3 z^{3}-285 z+59 z^{2}+254\right)(3 z-8 n-30) .
\end{aligned}
$$

We will prove by induction on $n \geq 0$ that $v_{n}(z) \in \mathbb{Z}[z]$. This is true for $n=$ $0,1,2$. Assume this is true up to $n+2$. The above recurrence then shows that $(3 z-8 n-14)(3 z-8 n-22) v_{n+3}(z) \in \mathbb{Z}[z]$. We now distinguish three cases.

- $n \equiv 0 \bmod 3$. By Gauss' lemma, there exists $r=a / b \in \mathbb{Q}$, with $(a, b)=1$, such that $r v_{n+3}(z) \in \mathbb{Z}[z]$ and $\frac{1}{r}(3 z-8 n-14)(3 z-8 n-22) \in \mathbb{Z}[z]$. This latter property implies that $a$ divides 3 because $8 n+14$ and $8 n+22$ don't belong to the same congruence class mod 3 . But the condition $n \equiv 0 \bmod 3$ implies that $a= \pm 1$ because $(8 n+14)(8 n+22) \not \equiv 0 \bmod 3$. Hence $v_{n+3}(z) \in \mathbb{Z}[z]$.

- $n \equiv 2 \bmod 3$. We have $(3 z-8 n-14)=3(z-d)$ with $d \in \mathbb{Z}$. But $\alpha(z) \in 3 \mathbb{Z}[z]$, $\beta(z) \in 3 \mathbb{Z}[z]$ and $n+1 \equiv 0 \bmod 3$; hence the right hand side of $(7.2)$ belongs to $3 \mathbb{Z}[z]$. Dividing both sides of (7.2) by 3 , we obtain that $(z-d)(3 z-8 n-22) v_{n+3}(z) \in$ $\mathbb{Z}[z]$. As above, Gauss' lemma implies that $v_{n+3}(z) \in \mathbb{Z}[z]$ because $8 n+22 \not \equiv 0$ $\bmod 3$.

- $n \equiv 1 \bmod 3$. We have $(3 z-8 n-22)=3(z-f)$ with $f \in \mathbb{Z}$. But again $\alpha(z) \in$ $3 \mathbb{Z}[z]$ and $n+2 \equiv 0 \bmod 3$; hence the right hand side of $(7.2)$ belongs to $3 \mathbb{Z}[z]$. Dividing both sides of (7.2) by 3 , we obtain that $(z-f)(3 z-8 n-14) v_{n+3}(z) \in \mathbb{Z}[z]$. Again, Gauss' lemma implies that $v_{n+3}(z) \in \mathbb{Z}[z]$ because $8 n+14 \not \equiv 0 \bmod 3$.

Thus in all cases, $v_{n+3}(z) \in \mathbb{Z}[z]$. This concludes the proof of the induction.

For the value of the degree, we again proceed by induction on $n$. The inital values $v_{0}, v_{1}, v_{2}$ are of the expected degrees. Let us assume that the degree of $v_{k}$ is $\leq k+1$ 
for any $k \leq n+2$. Then the degree of the right hand side of the above recurrence is clearly at most $n+6$. The left hand side $(3 z-8 n-14)(3 z-8 n-22) v_{n+3}(z)$ thus has degree at most $n+6$ and this finishes the induction.

7.4. Proof of Theorem 1, We only have to collect together the various bits contained in the propositions proved above, with one change: to get the recurrence (1.3) in the introduction from the recurrence (7.1) above, we change $U_{n}$ to $(n+1)^{2} U_{n}$ and accordingly $P_{n}, Q_{n}$ above to $(n+1)^{2} P_{n},(n+1)^{2} Q_{n}$. Some squares then disappear and the recurrence (7.1) takes the simpler form (1.3). With those notational changes, Point $(i)$ of Theorem 1 is the content of Proposition 17 proved in the previous section and point (ii) is a consequence of Propositions 14 and 16.

\section{Study of the Padé approximants in the Case $s=3$}

The method used in the previous sections can be generalised to provide (presumably) good rational approximations to $\Gamma^{\prime \prime}(1)$. However, as we shall see, this is not as simple as it seems.

8.1. Linear recurrence in the case $s=3$. It would be possible to proceed as in Section 6.2 where we first got the recurrence satisfied by the sequence $\left(A_{2, n}(z)\right)_{n \geq 0}$ and then showed that it was also satisfied by $\left(R_{j, 2, n}(z)\right)_{n \geq 0}, j=1,2$. However, this requires using the package MultInt for a triple integral representation of $A_{3, n}(z)$, which is not guaranteed to work in a reasonable time. Another approach is to carefully apply Zeilberger's program Ekhad 20] to the asymptotic expansion of $R_{1,3, n}(z)$ obtained in Proposition 4 , which has the advantage of being a single sum.

Proposition 18. The seven sequences $\left(A_{3, n}(z)\right)_{n \geq 0}, \quad\left(B_{j, 3, n}(z)\right)_{n \geq 0}$ and $\left(R_{j, 3, n}(z)\right)_{n \geq 0}, j=1,2,3$, are solutions of a fourth order recurrence of the form

$$
\alpha_{n}(z) u_{n+4}=\beta_{n}(z) u_{n+3}+\gamma_{n}(z) u_{n+2}+\delta_{n}(z) u_{n+1}+\kappa_{n}(z) u_{n},
$$

where $\alpha_{n}, \beta_{n}, \gamma_{n}, \delta_{n}, \kappa_{n}$ are polynomials in $\mathbb{Z}[n, z]$ of degree 8 in $n$ and of respective degrees $3,6,5,4$ and 3 in $z$.

Remark 10. The explicit expressions for the polynomial coefficients of the recurrence are huge and we prefer to give them only in the case $z=1$ (see Section 8.2). They can be found automatically by the procedure we will use during the proof.

Proof. Set $F(n, k)=\frac{1}{z^{k}} \frac{(k-n)_{n}^{3}}{n !^{3}} \Gamma(k)$, which is the term in the asymptotic expansion of $R_{1,3, n}(z)$. We note that $F(n, k)=0$ for $k=1, \ldots, n$.

The program Ekhad shows quickly that

$$
\begin{aligned}
& \alpha_{n}(z) F(n+4, k)-\beta_{n}(z) F(n+3, k)-\gamma_{n}(z) F(n+2, k) \\
& \quad-\delta_{n}(z) F_{0}(n+1, k, t)-\kappa_{n}(z, t) F(n, k)=G(n, k, z)-G(n, k+1, z)
\end{aligned}
$$

for some polynomials $\alpha_{n}(z), \beta_{n}(z), \gamma_{n}(z), \delta_{n}(z), \kappa_{n}(z)$ which are as described by the proposition and where $G(n, k, z) \in \mathbb{Q}(n, k, z)$ is of degree $-k+5$ in $z$, is defined for any $z \in \mathbb{C}^{\star}, n \geq 0, k \geq 0$ and has $(k-n-1)$ as a factor. This last fact means that $G(n, n+1, z)=0$ for any $n \geq 0$. 
We now sum both sides of (8.2) from $k=n+1$ to $k=N$ and get

$$
\begin{aligned}
\alpha_{n}(z) \sum_{k=n+1}^{N} F(n+4, k)-\beta_{n}(z) \sum_{k=n+1}^{N} F(n+3, k)-\gamma_{n}(z) \sum_{k=n+1}^{N} F(n+2, k) \\
-\delta_{n}(z) \sum_{k=n+1}^{N} F(n+1, k)-\kappa_{n}(z) \sum_{k=n+1}^{N} F(n, k)=\sum_{k=n+1}^{N}(G(n, k, z)-G(n, k+1, z)) \\
=G(n, n+1, z)-G(n, N+1, z)=-G(n, N+1, z)=\mathcal{O}\left(\frac{1}{z^{N-5}}\right) .
\end{aligned}
$$

Since $N$ can be arbitrarily large, the above equation means that the asymptotic expansion at $z=\infty$ of

$$
\begin{aligned}
J_{n}(z)=\alpha_{n}(z) R_{1,3, n+4}(z)- & \beta_{n}(z) R_{1,3, n+3}(z) \\
& -\gamma_{n}(z) R_{1,3, n+2}(z)-\delta_{n}(z) R_{1,3, n+1}(z)-\kappa_{n}(z) R_{1,3, n}(z)
\end{aligned}
$$

is the zero expansion. In other words, for any $k \geq 0$, we have $z^{k} J_{n}(z)=\mathcal{O}(1)$ as $z \rightarrow+\infty$. But by Proposition 4, we have

$$
R_{1,3, n+m}(z) \sim(-1)^{n} \frac{(n+m) !}{z^{n+m+1}} \quad \text { as } z \rightarrow \infty
$$

for any $m=0, \ldots, 4$; hence if $J_{n}(z)$ is not identically 0 , then we must have $\lim _{z \rightarrow \infty}\left|z^{k} J_{n}(z)\right|=+\infty$ for some integer $k \geq 0$. This contradicts what was found above and thus we have $J_{n}(z)=0$ for any $z \in \mathbb{C} \backslash[0,+\infty)$.

To show that $\left(A_{3, n}(z)\right)_{n \geq 0}$ satisfy the same recurrence, at least for $n$ large enough, we apply Proposition 7. (ii): this can be done because $\mathcal{E}_{1}(z) \notin \mathbb{C}(z)$ (see the end of Section 4.1). Then, we apply (i) of the same proposition to prove that $\left(R_{j, 3, n}(z)\right)_{n \geq 0}$ for $j=2,3$ are also solutions. Finally, it follows that $\left(B_{j, 3, n}(z)\right)_{n \geq 0}$, $j=1,2,3$ are solutions by linearity, at least for $n$ large enough. That the recurrence still holds for the small values of $n$ can be checked by direct computation.

8.2. Behavior of the solutions of recurrence (8.1), case $z=1$. The recurrence (8.1) for $z=1$ in Proposition 18 is

$$
\begin{gathered}
(8.3) \quad(n+3)(n+4)^{3}\left(729 n^{4}+5994 n^{3}+18297 n^{2}+24592 n+12290\right) U_{n+4} \\
=-(n+3)\left(2916 n^{7}+32724 n^{6}+99756 n^{5}-203447 n^{4}-2028202 n^{3}-5195409 n^{2}\right. \\
-5955904 n-2629472) U_{n+3}-\left(4374 n^{8}+123444 n^{7}+1407402 n^{6}+8701584 n^{5}+32365428 n^{4}\right. \\
\left.+74773104 n^{3}+105325246 n^{2}+82984580 n+28063902\right) U_{n+2}-(n+2)\left(2916 n^{7}+46575 n^{6}\right. \\
\left.+308088 n^{5}+1088368 n^{4}+2203128 n^{3}+2534133 n^{2}+1515768 n+355944\right) U_{n+1} \\
-(n+2)(n+1)^{3}\left(729 n^{4}+8910 n^{3}+40653 n^{2}+82084 n+61902\right) U_{n} .
\end{gathered}
$$

The Birkhoff-Trjitzinsky theory enables us to obtain the asymptotic behavior of its solutions. 
Proposition 19. The recurrence (8.3) admits four formal solutions whose leading terms as $n \rightarrow+\infty$ are:

$$
\begin{aligned}
& g_{1}(n)=\exp \left(4 e^{i \pi / 4} n^{3 / 4}+3 / 2 e^{i \pi / 2} n^{1 / 2}+5 / 8 e^{i 3 \pi / 4} n^{1 / 4}\right)\left(\frac{(-1)^{n}}{n^{3 / 2}}+\mathcal{O}\left(\frac{1}{n^{5 / 2}}\right)\right), \\
& g_{2}(n)=\exp \left(4 e^{-i \pi / 4} n^{3 / 4}+3 / 2 e^{-i \pi / 2} n^{1 / 2}+5 / 8 e^{-i 3 \pi / 4} n^{1 / 4}\right)\left(\frac{(-1)^{n}}{n^{3 / 2}}+\mathcal{O}\left(\frac{1}{n^{5 / 2}}\right)\right), \\
& g_{3}(n)=\exp \left(4 e^{3 i \pi / 4} n^{3 / 4}+3 / 2 e^{-i \pi / 2} n^{1 / 2}+5 / 8 e^{i \pi / 4} n^{1 / 4}\right)\left(\frac{(-1)^{n}}{n^{3 / 2}}+\mathcal{O}\left(\frac{1}{n^{5 / 2}}\right)\right), \\
& g_{4}(n)=\exp \left(4 e^{-3 i \pi / 4} n^{3 / 4}+3 / 2 e^{i \pi / 2} n^{1 / 2}+5 / 8 e^{-i \pi / 4} n^{1 / 4}\right)\left(\frac{(-1)^{n}}{n^{3 / 2}}+\mathcal{O}\left(\frac{1}{n^{5 / 2}}\right)\right) .
\end{aligned}
$$

From this result, we obtain upper bounds for the growth of the solutions of (8.3).

Proposition 20. All solutions of (8.3) are bounded in modulus by (a constant times)

and we also have

$$
\frac{1}{n^{3 / 2}} \exp \left(2 \sqrt{2} n^{3 / 4}-5 \sqrt{2} / 16 n^{1 / 4}\right)
$$

$$
\max \left(\left|R_{2,3, n}(1)\right|,\left|R_{3,3, n}(1)\right|\right) \ll \frac{1}{n^{3 / 2}} \exp \left(-2 \sqrt{2} n^{3 / 4}+5 \sqrt{2} / 16 n^{1 / 4}\right) .
$$

Proof. Proposition 19 shows that any solution grows at most like

$$
\left|g_{1}(n)\right|=\frac{1}{n^{3 / 2}} \exp \left(2 \sqrt{2} n^{3 / 4}-5 \sqrt{2} / 16 n^{1 / 4}\right)
$$

as $n \rightarrow+\infty$.

It is more difficult to find the exact behavior of a given sequence. We will prove below that $\left|R_{2,3, n}(1)\right|$ and $\left|R_{3,3, n}(1)\right|$ are bounded. Hence, they are automatically bounded by a constant times $\left|g_{2}(n)\right|=n^{-3 / 2} \exp \left(-2 \sqrt{2} n^{3 / 4}+(5 \sqrt{2} / 16) n^{1 / 4}\right)$.

An exact analogue of Lemma 1 for $\left|R_{2,3, n}(1)\right|$ and/or $\left|R_{3,3, n}(1)\right|$ is unknown, but we will use the same kind of idea. We start with the following identity: for any integer $s \geq 1$, we have

$$
\int_{0}^{\infty} \frac{\log (u)^{s-1}}{(1+u)(t+u)} \mathrm{d} u=\frac{\log (t)^{s}}{t-1}
$$

Hence,

$$
\begin{aligned}
& R_{2,3, n}(1)=\int_{0}^{\infty} \frac{1}{1+u} \int_{0}^{\infty} \frac{A_{3, n}(t)}{t+u} e^{-t} \mathrm{~d} t \mathrm{~d} u=\int_{0}^{\infty} \frac{R_{1,3, n}(-u)}{1+u} \mathrm{~d} u \\
& R_{3,3, n}(1)=\int_{0}^{\infty} \frac{\log (u)}{1+u} \int_{0}^{\infty} \frac{A_{3, n}(t)}{t+u} e^{-t} \mathrm{~d} t \mathrm{~d} u=\int_{0}^{\infty} \log (u) \frac{R_{1,3, n}(-u)}{1+u} \mathrm{~d} u
\end{aligned}
$$

(The interchange of the double integrals is justified by Fubini's theorem because both are absolutely convergent.)

By repeated integrations by parts, we get

$$
R_{1,3, n}(-u)=\int_{0}^{\infty} t^{n} e^{-t} \Phi_{n}(t, u) \mathrm{d} t
$$

for any $u>0$, where

$$
\Phi_{n}(t, u)=\frac{1}{n !^{2}} \frac{\mathrm{d}^{n}}{\mathrm{~d} t^{n}}\left(t^{n} \frac{\mathrm{d}^{n}}{\mathrm{~d} t^{n}}\left(\frac{t^{n}}{(t+u)^{n+1}}\right)\right) .
$$


Cauchy's theorem yields

$$
\Phi_{n}(t, u)=\frac{1}{(2 i \pi)^{2}} \int_{\mathcal{C}_{0} \times \widetilde{\mathcal{C}}_{0}} \frac{(z+w+t)^{n}(w+t)^{n}}{(z+w+t+u)^{n+1} z^{n+1} w^{n+1}} \mathrm{~d} z \mathrm{~d} w,
$$

where $\mathcal{C}_{0}$ and $\widetilde{\mathcal{C}_{0}}$ are circles centered at 0 such that $\mathcal{C}_{0} \times \widetilde{\mathcal{C}}_{0}$ does not meet $\{(z, w) \in$ $\mathbb{C} \times \mathbb{C}: z+w=-t-u\}$. Using the saddle point method, we get the estimate

$$
\begin{aligned}
\Phi_{n}(t, u) \sim \frac{c_{0}}{u^{1 / 3} n}\left(\frac{\left|t^{1 / 3}+u^{1 / 3}\right|^{5}}{\left(t^{1 / 3}+u^{1 / 3}\right)^{3 n+6}}+\frac{\left|t^{1 / 3}-e^{i \pi / 3} u^{1 / 3}\right|^{5}}{\left(t^{1 / 3}-e^{i \pi / 3} u^{1 / 3}\right)^{3 n+6}}\right. \\
\left.+\frac{\left|t^{1 / 3}-e^{-i \pi / 3} u^{1 / 3}\right|^{5}}{\left(t^{1 / 3}-e^{-i \pi / 3} u^{1 / 3}\right)^{3 n+6}}\right)
\end{aligned}
$$

as $n \rightarrow+\infty$, where the constant $c_{0}$ is independent of $n, t, u$. (We skip the technical details of this computation.) We observe that for any $t \geq 0, u \geq 0$, the moduli of the three functions

$$
\frac{t^{n}}{\left(t^{1 / 3}+u^{1 / 3}\right)^{3 n}}, \quad \frac{t^{n}}{\left(t^{1 / 3}-e^{i \pi / 3} u^{1 / 3}\right)^{3 n}}, \quad \frac{t^{n}}{\left(t^{1 / 3}-e^{-i \pi / 3} u^{1 / 3}\right)^{3 n}}
$$

are bounded by an absolute constant $c_{1}$ independent of $n$.

Remembering the expressions (8.4), it follows that there exists an absolute constant $c_{0}>0$ independent of $n$ such that, for $j=1,2$,

$$
\begin{aligned}
\left|R_{j+1,3, n}(1)\right| \leq c_{0} \int_{0}^{\infty} \int_{0}^{\infty} \frac{e^{-t} \ln (u)^{j-1}}{(1+u) u^{1 / 3}}\left(\frac{1}{\left|t^{1 / 3}+u^{1 / 3}\right|}\right. \\
\left.+\frac{1}{\left|t^{1 / 3}-e^{i \pi / 3} u^{1 / 3}\right|}+\frac{1}{\left|t^{1 / 3}-e^{i \pi / 3} u^{1 / 3}\right|}\right) \mathrm{d} t \mathrm{~d} u .
\end{aligned}
$$

Since the integral on the right hand sides converges, we have proved that $\left(R_{2,3, n}(1)\right)_{n \geq 0}$ and $\left(R_{3,3, n}(1)\right)_{n \geq 0}$ are bounded sequences.

\section{RAtional approximations for $\Gamma^{\prime \prime}(1)-2 \Gamma^{\prime}(1)^{2}$}

To construct rational "approximations" of $\Gamma^{\prime \prime}(1)=\zeta(2)+\gamma^{2}$, we set $s=3$ and $z=1$ in the Padé approximants studied in Sections 4.3 and 8 . We showed that $R_{j, 3, n}(z)=A_{3, n}(z) \mathcal{E}_{j}(z)+B_{j, 3, n}(z)$ and $B_{j, 3, n}(z)=\sum_{k=1}^{j}\left(\begin{array}{c}j-1 \\ k-1\end{array}\right) \Gamma^{(j-k)}(1) \widetilde{B}_{k, 3, n}(z)$ for some polynomial $\widetilde{B}_{j, 3, n}(z), j=1,2,3$, which clearly all satisfy the recurrence (8.1). When $z=1$ and $j=2,3$, we obtain that

$$
\begin{aligned}
& R_{3,3, n}(1)=A_{3, n}(1) \mathcal{E}_{3}(1)+\widetilde{B}_{1,3, n}(1) \Gamma^{\prime \prime}(1)+2 \widetilde{B}_{2,3, n}(1) \Gamma^{\prime}(1)+\widetilde{B}_{3,3, n}(1) \\
& R_{2,3, n}(1)=A_{3, n}(1) \mathcal{E}_{2}(1)+\widetilde{B}_{1,3, n}(1) \Gamma^{\prime}(1)+\widetilde{B}_{2,3, n}(1)
\end{aligned}
$$

By the method used in the proof of Proposition 9] it is not difficult to provide explicit expressions for the polynomials $A_{3, n}(z)$ and $\widetilde{B}_{j, 3, n}(z), j=1,2,3$.

Proposition 21. Let

$$
b_{k, n}=\frac{(-1)^{k}}{k !} \sum_{j=0}^{k} \sum_{\ell=0}^{j}\left(\begin{array}{c}
n \\
k-j
\end{array}\right)\left(\begin{array}{c}
n+j \\
n
\end{array}\right)\left(\begin{array}{c}
n \\
\ell
\end{array}\right)\left(\begin{array}{c}
n \\
j-\ell
\end{array}\right)\left(\begin{array}{c}
n+\ell \\
n
\end{array}\right) .
$$


Then we have

$$
\begin{aligned}
A_{3, n}(z) & =\sum_{k=0}^{3 n} b_{k, n} z^{k}, \quad \widetilde{B}_{1,3, n}(z)=\sum_{k=0}^{3 n} b_{k, n} \sum_{j=0}^{k-1} j ! z^{k-1-j}, \\
\widetilde{B}_{2,3, n}(z) & =\sum_{k=0}^{3 n} b_{k, n} z^{k} \sum_{j=0}^{k-1} j ! H_{j} z^{k-1-j}, \quad \widetilde{B}_{3,3, n}(z)=\sum_{k=0}^{3 n} b_{k, n} \sum_{j=0}^{k-1} j ! H_{j}^{[2]} z^{k-1-j} .
\end{aligned}
$$

The degree of the polynomials is at most $3 n$ and the four polynomials belong to $\frac{1}{(3 n) !} \mathbb{Z}[z]$.

These explicit expressions can be used to compute the approximations we will construct in the next sections and which are at the heart of Theorem 2, The last assertion, which is probably not optimal, is a consequence of the fact that $(3 n) ! b_{k, n}$, $j ! H_{j}$ and $j ! H_{j}^{[2]}$ are integers.

9.1. Determinants related to $\zeta(2)-\gamma^{2}$ and $\gamma$. It is possible to cancel out $\mathcal{E}_{3}(1)$ and $\Gamma^{\prime}(1)$ by considering determinants based on $R_{3,3, n}(1), R_{3,3, n+1}(1)$ and $R_{3,3, n+2}(1)$. More precisely, the method used in the proof of Proposition 14 shows that

$$
\begin{aligned}
& \left|\begin{array}{lll}
A_{3, n} & \widetilde{B}_{2,3, n} & R_{3,3, n} \\
A_{3, n+1} & \widetilde{B}_{2,3, n+1} & R_{3,3, n+1} \\
A_{3, n+2} & \widetilde{B}_{2,3, n+2} & R_{3,3, n+2}
\end{array}\right| \\
& \quad=\left|\begin{array}{lll}
A_{3, n} & \widetilde{B}_{2,3, n} & \widetilde{B}_{1,3, n} \\
A_{3, n+1} & \widetilde{B}_{2,3, n+1} & \widetilde{B}_{1,3, n+1} \\
A_{3, n+2} & \widetilde{B}_{2,3, n+1} & \widetilde{B}_{1,3, n+1}
\end{array}\right| \Gamma^{\prime \prime}(1)-\left|\begin{array}{lll}
A_{3, n} & \widetilde{B}_{3,3, n} & \widetilde{B}_{2,3, n} \\
A_{3, n+1} & \widetilde{B}_{3,3, n+1} & \widetilde{B}_{2,3, n+1} \\
A_{3, n+2} & \widetilde{B}_{3,3, n+1} & \widetilde{B}_{2,3, n+1}
\end{array}\right| .
\end{aligned}
$$

(We removed the argument $z=1$ for better readability.) Unfortunately, this sequence of linear forms in $\mathbb{Q} \Gamma^{\prime \prime}(1)+\mathbb{Q}$ does not seem to tend to 0 as $n \rightarrow+\infty$ and, more surprisingly, the quotient of the two determinants on the right hand side does not seem to tend to $\Gamma^{\prime \prime}(1)$.

Another possibility is to work with $R_{2,3, n}(1)$ and $R_{2,3, n}(1)$ simultaneously. This idea leads to the construction of $2 \times 2$ determinants that will enable us to approximate $\Gamma^{\prime \prime}(1)-2 \Gamma^{\prime}(1)^{2}$. For simplicity, we set $R_{n}=R_{3,3, n}(1)-2 \Gamma^{\prime}(1) R_{2,3, n}(1)$, $A_{n}=A_{3, n}(1), B_{n}=\widetilde{B}_{1,3, n}(1), C_{n}=\widetilde{B}_{2,3, n}(1)$ and $D_{n}=\widetilde{B}_{3,3, n}(1)$.

The linear combination (9.1) $-2 \Gamma^{\prime}(1)^{2} \cdot(9.2)$ reads

$$
R_{n}=A_{n}\left(\mathcal{E}_{3}(1)-2 \Gamma^{\prime}(1) \mathcal{E}_{2}(1)\right)+B_{n}\left(\Gamma^{\prime \prime}(1)-2 \Gamma^{\prime}(1)^{2}\right)+D_{n},
$$

and the determinant $\rho_{n}=\left|\begin{array}{ll}R_{n} & A_{n} \\ R_{n+1} & A_{n+1}\end{array}\right|$ is such that $\rho_{n}=q_{n}\left(\Gamma^{\prime \prime}(1)-2 \Gamma^{\prime}(1)^{2}\right)-$ $p_{n} \in \mathbb{Q}\left(\zeta(2)-\gamma^{2}\right)+\mathbb{Q}$, where $q_{n}=\left|\begin{array}{ll}B_{n} & A_{n} \\ B_{n+1} & A_{n+1}\end{array}\right|$ and $p_{n}=\left|\begin{array}{ll}A_{n} & D_{n} \\ A_{n+1} & D_{n+1}\end{array}\right|$.

Similarly, set $\widehat{\rho}_{n}=\left|\begin{array}{ll}R_{2,3, n} & A_{n} \\ R_{2,3, n+1} & A_{n+1}\end{array}\right|$. Then, from (9.2), we deduce that $\widehat{\rho}_{n}=$ $q_{n} \Gamma^{\prime}(1)+\widehat{p}_{n}=-q_{n} \gamma+\widehat{p}_{n} \in \mathbb{Q} \gamma+\mathbb{Q}$, where $\widehat{p}_{n}=\left|\begin{array}{ll}C_{n} & A_{n} \\ C_{n+1} & A_{n+1}\end{array}\right|$.

Hence, we have constructed simultaneous rational "approximations" to the numbers $\zeta(2)-\gamma^{2}$ and $\gamma$ with common denominator $q_{n}$. It remains to prove that they are really approximations and to find the rate of convergence towards these numbers. 
9.2. Linear recurrence for $\zeta(2)-\gamma^{2}$ and $\gamma$. To find the recurrence satisfied by the sequences defined in the previous section, we can apply Proposition 5.5 to the recurrence (8.3) written as $U_{n+4}=s_{n} U_{n+3}+p_{n} U_{n+2}+q_{n} U_{n+1}+r_{n} U_{n}$.

Proposition 22. The sequences $\left(p_{n}\right)_{n \geq 0},\left(\widehat{p}_{n}\right)_{n \geq 0},\left(q_{n}\right)_{n \geq 0},\left(\rho_{n}\right)_{n \geq 0}$ and $\left(\widehat{\rho}_{n}\right)_{n \geq 0}$ satisfy a linear recurrence $\mathfrak{R}: \sum_{j=0}^{6} \alpha_{j}(n) U_{n+j}=0$ of order 6 , where the $\alpha_{j}(n)$ are polynomials of degree 28 with integer coefficients.

Furthermore, $(3 n+3) !(3 n) ! p_{n},(3 n+3) !(3 n) ! \widehat{p}_{n}$ and $(3 n+3) !(3 n) ! q_{n}$ are integers.

The coefficients of the recurrence $\mathfrak{R}$ are huge and are given in the Annex to this paper. We will use in the next section the fact that $\alpha_{6}(n)=(n+7)^{3}(n+6)^{4}$ $(n+5)^{2} g(n)$ and $\alpha_{0}(n)=(n+3)^{2}(n+2)^{4}(n+1)^{3} g(n-1)$ for some polynomial $g(n)$ also given explicitly in the Annex.

The integrality assertion follows from Proposition 21.

9.3. Asymptotic behavior of the solutions of $\mathfrak{R}$. In spite of the complicated form of its coefficients, it is not difficult to study $\mathfrak{R}$ on a computer. In particular, Birkhoff-Trjitzinsky theory provides the asymptotic behavior of its solutions.

Proposition 23. The recurrence $\mathfrak{R}$ admits six formal solutions whose leading terms as $n \rightarrow+\infty$ are

$$
\begin{aligned}
& \widetilde{g}_{1}(n)=\exp \left(4 \sqrt{2} n^{3 / 4}-5 \sqrt{2} / 8 n^{1 / 4}\right)\left(\frac{1}{n^{27 / 8}}+\mathcal{O}\left(\frac{1}{n^{35 / 8}}\right)\right), \\
& \widetilde{g}_{2}(n)=\exp \left(-4 \sqrt{2} n^{3 / 4}+5 \sqrt{2} / 8 n^{1 / 4}\right)\left(\frac{1}{n^{27 / 8}}+\mathcal{O}\left(\frac{1}{n^{35 / 8}}\right)\right), \\
& \widetilde{g}_{3}(n)=\exp \left(4 \sqrt{2} i n^{3 / 4}+5 \sqrt{2} / 8 i n^{1 / 4}\right)\left(\frac{1}{n^{27 / 8}}+\mathcal{O}\left(\frac{1}{n^{35 / 8}}\right)\right), \\
& \widetilde{g}_{4}(n)=\exp \left(-4 \sqrt{2} i n^{3 / 4}-5 \sqrt{2} / 8 i n^{1 / 4}\right)\left(\frac{1}{n^{27 / 8}}+\mathcal{O}\left(\frac{1}{n^{35 / 8}}\right)\right), \\
& \widetilde{g}_{5}(n)=\exp \left(i 3 n^{1 / 2}\right)\left(\frac{1}{n^{11 / 4}}+\mathcal{O}\left(\frac{1}{n^{15 / 4}}\right)\right), \\
& \widetilde{g}_{6}(n)=\exp \left(-i 3 n^{1 / 2}\right)\left(\frac{1}{n^{11 / 4}}+\mathcal{O}\left(\frac{1}{n^{15 / 4}}\right)\right) .
\end{aligned}
$$

We remark that one solution tends to infinity, one solution tends to 0 quickly whereas the last four decrease slowly to 0 .

It is not easy to find precisely the behavior of the sequences we are interested in, but we can at least prove the following bounds.

Proposition 24. There exist two constants $c_{0}>0$ and $c_{1} \neq 0$ such that

$$
\left|\rho_{n}\right| \leq \frac{c_{0}}{n^{27 / 8}}, \quad\left|\widehat{\rho}_{n}\right| \leq \frac{c_{0}}{n^{27 / 8}}, \quad q_{n} \sim \frac{c_{1}}{n^{27 / 8}} \exp \left(4 \sqrt{2} n^{3 / 4}-5 \sqrt{2} / 8 n^{1 / 4}\right) .
$$

Remark 11. We did not try to find an equivalence for $\rho_{n}$ and $\widehat{\rho}_{n}$, but, numerically, both do not seem to decrease like $\widetilde{g}_{2}(n)$.

Proof. By Proposition 20, we know that $\left|\rho_{n}\right| \ll n^{-3}$ and $\left|\widehat{\rho}_{n}\right| \ll n^{-3}$. Hence $\rho_{n}$ and $\widehat{\rho}_{n}$ tend to 0 at least like $\left|\widetilde{g}_{3}(n)\right|=n^{-27 / 8}$. 
The case of $q_{n}$ is more complicated. Let us define the determinant

$$
\widetilde{\rho}_{n}=\left|\begin{array}{ll}
B_{n} & R_{n} \\
B_{n+1} & R_{n+1}
\end{array}\right|=-q_{n} \mathcal{E}_{3}(1)+u_{n}, \text { where } u_{n}=\left|\begin{array}{ll}
B_{n} & D_{n} \\
B_{n+1} & D_{n+1}
\end{array}\right| .
$$

We will prove below that the sequences $\left(q_{n}\right)_{n \geq 0},\left(p_{n}\right)_{n \geq 0},\left(\widehat{p}_{n}\right)_{n \geq 0}$ and $\left(u_{n}\right)_{n \geq 0}$ are linearly independent over $\mathbb{C}$. Assuming this, at least one of them must tend to infinity by the Birkhoff-Trjitzinsky theory. Furthermore, as for $\rho_{n}$ and $\widehat{\rho}_{n}$, it is clear that $\widetilde{\rho}_{n}$ tends to 0 ; hence, if $q_{n}$ tends to 0 , this is also the case of $p_{n}, \widehat{p}_{n}$ and $u_{n}$, which is a contradiction.

Therefore, $\left(q_{n}\right)_{n \geq 0}$ tends to infinity with $n$ and the only possibility is that it increases like $\widetilde{g}_{1}(n)$; i.e., there exists a constant $c_{1} \neq 0$ such that

$$
q_{n} \sim \frac{c_{1}}{n^{27 / 8}} \exp \left(4 \sqrt{2} n^{3 / 4}-5 \sqrt{2} / 8 n^{1 / 4}\right) .
$$

It remains to prove that the sequences $\left(q_{n}\right)_{n \geq 0},\left(p_{n}\right)_{n \geq 0},\left(\widehat{p}_{n}\right)_{n \geq 0}$ and $\left(u_{n}\right)_{n \geq 0}$ are independent. We will prove more. Let us introduce two further determinants:

$$
v_{n}=\left|\begin{array}{ll}
B_{n} & C_{n} \\
B_{n+1} & C_{n+1}
\end{array}\right|, \quad w_{n}=\left|\begin{array}{ll}
C_{n} & D_{n} \\
C_{n+1} & D_{n+1}
\end{array}\right| .
$$

The six determinants $p_{n}, q_{n}, \widehat{p}_{n}, u_{n}, v_{n}, w_{n}$ are solutions of the recurrence $\mathfrak{R}$, and we are going to show that they form a basis over $\mathbb{C}$ of its solutions. For this, it is enough to prove that the $6 \times 6$ determinant $\Phi_{n}$ whose lines are the vectors $\left(p_{n+j}\right)_{j=0, \ldots, 5}, \ldots,\left(z_{n+j}\right)_{j=0, \ldots, 5}$ is non-zero for large $n$. But, we have that $\Phi_{n}=$ $(-1)^{n-1} \Phi_{1} \times \prod_{k=1}^{n-1} \frac{p_{0}(k)}{p_{6}(k)}$, where $p_{0}(n)$ and $p_{6}(n)$ are the "extremal" coefficients of $\mathfrak{R}$ explicitly given at the end of Section 9.2 in term of a polynomial $g$. We find that

$$
\left|\Phi_{n}\right|=\frac{2^{35} 3^{16} 5^{11} 7^{3} \cdot 542415636464476283}{(n+1)^{3}(n+2)^{7}(n+3)^{9}(n+4)^{9}(n+5)^{7}(n+6)^{3} g(n-1)}\left|\Phi_{1}\right|
$$

and $\left|\Phi_{1}\right|=\frac{542415636464476283}{2^{18} 3^{12} 5^{7} 7^{3}}$. Hence, for large $n$, we have $\Phi_{n} \neq 0$, which concludes the proof of the proposition.

9.4. Proof of Theorem 2. As in the case of Theorem 1 we just have to put the pieces together. Set $a_{1, n}=(n+1)^{3} \widehat{p}_{n}, a_{2, n}=(n+1)^{3} p_{n}$ and $b_{n}=(n+1)^{3} q_{n}$, which all belong to $\frac{1}{(3 n+2) !(3 n) !} \mathbb{Z}$ by Proposition 22 This proposition also shows that these sequences are solutions of a recurrence (1.6) as stated in Theorem 2 which is a simplified version of $\mathfrak{R}$, where some cube factors have disappeared (see the Annex). The asymptotic behavior of the sequences follows from Proposition 24

\section{Annex: Coefficients of the RECURREnce $\mathfrak{R}$}

We provide here the initial values of the sequences $\left(a_{1, n}\right)_{n \geq 0},\left(a_{2, n}\right)_{n \geq 0}$ and $\left(b_{n}\right)_{n \geq 0}$ introduced in Theorem 2 as well as the coefficients of the sixth order recurrence $\mathfrak{R}: \sum_{j=0}^{6} \alpha_{j}(n) U_{n+j}=0$ satisfied by the sequences $\left(\widehat{p}_{n}\right)_{n \geq 0},\left(p_{n}\right)_{n \geq 0}$ and $\left(q_{n}\right)_{n \geq 0}$ introduced in Section 9.1. We recall $a_{1, n}=(n+1)^{3} \widehat{p}_{n}, a_{2, n}=(n+1)^{3} p_{n}$ 
and $b_{n}=(n+1)^{3} q_{n}$. We have

$$
\begin{aligned}
a_{1, n} & =-2,29, \frac{5885}{4}, \frac{1130135}{27}, \frac{57594501}{64}, \frac{54102373562}{3375}, \\
a_{2, n} & =-2,84, \frac{13335}{4}, \frac{3424463}{36}, \frac{14135602661}{6912}, \frac{52459002112643}{1440000}, \\
b_{n} & =-1,51, \frac{10177}{4}, \frac{2610521}{36}, \frac{299338717}{192}, \frac{399914313019}{14400} .
\end{aligned}
$$

We have $a_{1, n} / b_{n} \rightarrow \gamma$ and $a_{2, n} / b_{n} \rightarrow \zeta(2)-\gamma^{2}$ as $n \rightarrow+\infty$.

We have $\alpha_{6}(n)=(n+7)^{3}(n+6)^{4}(n+5)^{2} g(n)$ and $\alpha_{0}(n)=(n+3)^{2}(n+2)^{4}$ $(n+1)^{3} g(n-1)$, where

$$
\begin{aligned}
& g(n)=83682825624 n^{19}+5215067202429 n^{18}+152458227391932 n^{17} \\
& +2777470002640620 n^{16}+35325735571692396 n^{15}+332981886215308977 n^{14} \\
& +2409616397398674420 n^{13}+13679321195302120800 n^{12}+61706325831305809608 n^{11} \\
& +222559825069576726155 n^{10}+642164667745863871620 n^{9} \\
& +1474453073521538662412 n^{8}+2660820887533978586924 n^{7} \\
& +3688762952280998863283 n^{6}+3768165799741173406988 n^{5} \\
& +2601927871824904518632 n^{4}+936452965112136997448 n^{3} \\
& -111682802237940807724 n^{2}-260863346815372837120 n-79358069872714705024 .
\end{aligned}
$$

The five other coefficients follow.

$$
\begin{aligned}
\alpha_{5}(n)= & -(n+5)^{2}(n+6)^{3}\left(502096953744 n^{23}+47273822908758 n^{22}+2092789693027584 n^{21}\right. \\
+ & 58015237795632504 n^{20}+1131077306383536672 n^{19}+16506560669364758826 n^{18} \\
+187324948229909087232 n^{17}+1694886740772551389449 n^{16}+12433106089743379511616 n^{15} & +74778447249095109798582 n^{14}+371344332872338942100616 n^{13} \\
& +1528015101398154070637034 n^{12}+5212036196627818871609688 n^{11} \\
+ & 14695949275426642308113646 n^{10}+34030999203439611543890304 n^{9} \\
+ & 63992524960553242428422841 n^{8}+95931896009758745043893064 n^{7} \\
+ & 111186664542020448915219772 n^{6}+94148331863956910914875640 n^{5} \\
& +50972087616381261858826828 n^{4}+9143558678753028780989776 n^{3} \\
& -9136223070830414842846064 n^{2}-7300240129419137615929856 n \\
& -1767373066831337541843456),
\end{aligned}
$$




$$
\begin{aligned}
& \alpha_{4}(n)=(n+5)^{3}\left(1255242384360 n^{25}+109439701994187 n^{24}+4507485520683312 n^{23}\right. \\
& +116464555712558538 n^{22}+2113029301778202600 n^{21}+28523911803790485537 n^{20} \\
& +295670887621994238804 n^{19}+2387026781742108138786 n^{18}+14961881372754629129688 n^{17} \\
& +70479354522862285539210 n^{16}+220628690628242306979276 n^{15} \\
& +165675557916168459340062 n^{14}-3110843673701850213533736 n^{13} \\
& -24457229872723298409747471 n^{12}-114182963654122277648822980 n^{11} \\
& -395865026342983204883963306 n^{10}-1081703758545008286382351272 n^{9} \\
& -2378573759652537959838451027 n^{8}-4230000289863354871845360140 n^{7} \\
& -6056446994844253022398232784 n^{6}-6893387355339798817347909176 n^{5} \\
& -6099994965404364718704482596 n^{4}-4047646867961219357643431232 n^{3} \\
& -1895233044384779296961010976 n^{2}-558363716355809256087087104 n \\
& -77835199837793348629724160), \\
& \alpha_{3}(n)=-(n+4)^{3}\left(1673656512480 n^{25}+130243019992020 n^{24}+4641048992546388 n^{23}\right. \\
& +98548235320764627 n^{22}+1332717826676092380 n^{21}+10512649049974299774 n^{20} \\
& +9957833272423695996 n^{19}-1013766109194744817461 n^{18}-16772944094332329078648 n^{17} \\
& -167704080208998118841286 n^{16}-1240169152914351165178836 n^{15} \\
& -7226464342726090616836460 n^{14}-34133063958195859418085124 n^{13} \\
& -132558211151584919103170286 n^{12}-426126071741293205743109664 n^{11} \\
& -1135842894967603634175667881 n^{10}-2504560039067611217162572236 n^{9} \\
& -4539867842503552572392991142 n^{8}-6692112093905239454389694900 n^{7} \\
& -7889806620359002949998388109 n^{6}-7255122308457723459409050628 n^{5} \\
& -5004771074477163991233072440 n^{4}-2427130458798950391053978904 n^{3} \\
& -730143340903879677133231036 n^{2}-97079670872378138528993024 n \\
& +2888284259604646368585600) \\
& \alpha_{2}(n)=(n+3)^{3}\left(1255242384360 n^{25}+109021287866067 n^{24}+4476088189413774 n^{23}\right. \\
& +115449674036040495 n^{22}+2096428336751729628 n^{21}+28459359782620402914 n^{20} \\
& +299224149269524225956 n^{19}+2489581604671304610924 n^{18}+16591882794957454092480 n^{17} \\
& +88987140061743491823096 n^{16}+383015809017980948531664 n^{15} \\
& +1307342993187170443205442 n^{14}+3449882707220123181299220 n^{13} \\
& +6715502395297058098778883 n^{12}+9050884636200561121581082 n^{11} \\
& +10473683996521005943885043 n^{10}+34976892905602880094614556 n^{9} \\
& +169371127360634348300394540 n^{8}+562323815799789656729159112 n^{7} \\
& +1290308358544698937289767692 n^{6}+2133934920517610212247675376 n^{5} \\
& +2572948232492602076990966640 n^{4}+2225440901784395089632523392 n^{3} \\
& +1315639138004063783827741824 n^{2}+477890041746520637009571840 n \\
& +80652658347913045992960000)
\end{aligned}
$$




$$
\begin{aligned}
\alpha_{1}(n)=- & (n+3)^{2}(n+2)^{3}\left(502096953744 n^{23}+48947479421238 n^{22}+2259955435303260 n^{21}\right. \\
+ & 65765526565004154 n^{20}+1354021086721570704 n^{19}+20986039777254988176 n^{18} \\
+254343854243707457220 n^{17}+2471440558772566757997 n^{16}+19584114363729419446620 n^{15} & \\
& +128036881467882649984482 n^{14}+695970530175526138291536 n^{13} \\
& +3159890793789534406161804 n^{12}+12006859882829922244786716 n^{11} \\
& +38163425164615328756953428 n^{10}+101166194726777877489926608 n^{9} \\
& +222395888495179811601367145 n^{8}+401846922491417937305961144 n^{7} \\
& +589110060671213666440210564 n^{6}+687775891586369125568288528 n^{5} \\
& +622430627582169970723265508 n^{4}+419272360294060293370554496 n^{3} \\
& +196873874819353464216975584 n^{2}+57119262537702140622994944 n \\
& +7635535812462194001878400)
\end{aligned}
$$

\section{ACKNowledgement}

The author warmly thanks the referee for her/his very detailed report that helped to improve the initial version of this article.

\section{REFERENCES}

[1] K. Alladi and M. Robinson, Legendre polynomials and irrationality, J. Reine Angew. Math. 318 (1980), 137-155. MR 579389 (81i:10036)

[2] Y. André, Arithmetic Gevrey series and transcendence. A survey, J. Théor. Nombres Bordeaux 15 (2003), no. 1, 1-10. MR2018997 (2004m:11115)

[3] G. E. Andrews, R. A. Askey and R. Roy, Special Functions, The Encyclopedia of Mathematics and Its Applications, vol. 71, (G.-C. Rota, ed.), Cambridge University Press, Cambridge (1999). MR1688958 (2000g:33001)

[4] R. Apéry, Irrationalité de $\zeta(2)$ et $\zeta(3)$, Astérisque 61 (1979), 11-13.

[5] A. I. Aptekarev, A. Branquinho and W. van Assche, Multiple orthogonal polynomials for classical weights, Trans. Amer. Math. Soc. 355 (2003), 3887-3914. MR1990569(2004g:33014)

[6] A. I. Aptekarev (editor), Rational approximants for Euler constant and recurrence relations, Sovremennye Problemy Matematiki ("Current Problems in Mathematics"), vol. 9, MIAN (Steklov Institute), Moscow, 2007.

[7] G. D. Birkhoff and W. J. Trjitzinsky, Analytic theory of singular difference equations, Acta Math. 60 (1932), 1-89. MR1555364

[8] H. Cohen, Accélération de la convergence de certaines récurrences linéaires, Sémin. Théor. Nombres 1980-1981, Exposé no.16, 2 pp. (1981).

[9] C. Elsner, On a sequence transformation with integral coefficients for Euler's constant, Proc. Amer. Math. Soc. 123 (1995), no. 3, 1537-1541. MR1233969 (95f:11111)

[10] S. Fischler and T. Rivoal, Un exposant de densité en approximation rationnelle, Internat. Math. Res. Notices 2006 (2006), Article ID 95418, 48 pages. MR2272100 (2007h:11079)

[11] G. H. Hardy, Divergent series, Second edition, Chelsea Publishing Company, 1991. MR.1188874 (93g:01100)

[12] M. Hata, Rational approximations to $\pi$ and some other numbers, Acta. Arith. 63 (1993), 335-349. MR1218461 (94e:11082)

[13] M. Kontsevich and D. Zagier, Periods, in Mathematics Unlimited - 2001 and Beyond, Springer, Berlin, 2001, 771-808. MR.1852188 (2002i:11002)

[14] C. Krattenthaler and T. Rivoal, How can we escape Thomae's relations?, J. Math. Soc. Japan 58 (2006), no. 1, 183-210. MR2204570 (2007b:11106)

[15] J.-P. Ramis, Séries divergentes et théories asymptotiques, Bull. Soc. Math. France 121 (1993), Panoramas et Synthèses, 74 pp. MR:1272100 (95h:34074) 
[16] T. Rivoal, Simultaneous polynomial approximations of the Lerch function, Preprint (2007), 15 pages, to appear in Canadian J. Math.

[17] T. Rivoal and W. Zudilin, Diophantine properties of numbers related to Catalan's constant, Math. Annalen 326 (2003), no. 4, 705-721. MR2003449 (2004k:11119)

[18] A. Tefara, MultInt, a Maple Package for Multiple Integration by the WZ Method, J. Symb. Comp. 34 (2002), no. 5, 329-353. MR1937465 (2004b:33030) The package Multint is available here: http://faculty.gvsu.edu/teferaa/html/MultInt.mpl.

[19] M. Waldschmidt, Valeurs zêta multiples. Une introduction, J. Théor. Nombres Bordeaux 12 (2000), no. 2, 581-595. MR.1823204(2002a:11106)

[20] H. S. Wilf and D. Zeilberger, An algorithmic proof theory for hypergeometric (ordinary and "q") multisum/integral identities, Invent. Math. 108 (1992), no. 3, 575-633. MR.1163239 (93k:33010)

[21] J. Wimp and D. Zeilberger, Resurrecting the asymptotics of linear recurrences, J. Math. Anal. Appl. 111 (1985), no. 1, 162-176. MR808671 (87b:05015)

[22] D. Zeilberger, A holonomic systems approach to special functions identities, J. Comput. Appl. Math. 32 (1990), no. 3, 321-368. MR1090884 (92b:33014)

[23] W. Zudilin, On third-order Apéry-like recursion for $\zeta(5)$, Mat. Zametki 72 (2002), no. 5, 796-800 (Russian); English transl., Math. Notes 72 (2002), no. 5-6, 733-737. MR.1963141 (2004d:11061)

Institut Fourier, CNRS UMR 5582, Université Grenoble 1, 100 Rue Des Maths, BP 74, 38402 Saint-Martin d'Hères Cedex, France

URL: http://www-fourier.ujf-grenoble.fr/ ${ }^{\sim}$ rivoal 